\title{
Scissors Congruence, the Golden Ratio and Volumes in Hyperbolic 5-Space
}

\author{
Ruth Kellerhals
}

Received: 12 September 2011 / Accepted: 17 January 2012 / Published online: 8 February 2012

(C) Springer Science+Business Media, LLC 2012

\begin{abstract}
By different scissors congruence techniques a number of dissection identities are presented between certain quasi-Coxeter polytopes, whose parameters are related to the golden section, and an ideal regular simplex in hyperbolic 5-space. As a consequence, several hyperbolic polyhedral 5-volumes can be computed explicitly in terms of Apéry's constant $\zeta(3)$ and the trilogarithmic value $J_{3}\left(\frac{\pi}{5}\right)$.
\end{abstract}

Keywords Scissors congruence - Hyperbolic 5-space - Quasi-Coxeter polytopes · Trilogarithms · Zeta values

\section{Introduction}

Polytopes in hyperbolic space $\mathbb{H}^{n}$ are important objects in the study of hyperbolic space forms and arise in various contexts ranging from geometry and topology to mathematical physics. They serve as combinatorial models and allow us sometimes to compute geometric-topological data such as Betti numbers, Euler characteristic, systole and volume. These quantities are related by (in-)equalities that allow us to concentrate on the most important quantity, the volume of a hyperbolic polytope.

In contrast to lower dimensions, polyhedral volume in hyperbolic 5-space is only partially understood but forms the higher dimensional doorway in view of Schläfli's volume differential formula (see e.g. [9, 12], [16, p. 118]). In [10], we provide, for a certain two parameter-family of doubly asymptotic orthoschemes a comparatively simple formula in terms of their dihedral angles and the so-called trilobachevsky function $\pi_{3}(\omega)$. As a consequence, the covolume of the hyperbolic Coxeter group

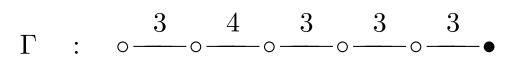

R. Kellerhals $(\varangle)$

Department for Mathematics, University of Fribourg, 1700 Fribourg, Switzerland e-mail: Ruth.Kellerhals@unifr.ch 
could be computed and equals $7 \zeta(3) / 46080$, allowing to identify the (arithmetic) quotient orbifold $Q=\mathbb{H}^{5} / \Gamma$ as the (unique) cusped hyperbolic 5-space form of minimal volume (see [7]; the bullet indicates the occurrence of one cusp in $Q$ ). In [11], we completely solved the volume problem for hyperbolic 5-space by deriving a volume formula for the generators of the scissors congruence group $\mathcal{P}\left(\overline{\mathbb{H}^{5}}\right)$ in terms of (many) trilogarithm expressions. In practice, however, this formula requires the dissection/extension of a given polytope $P \subset \overline{\mathbb{H}^{5}}$ by doubly asymptotic orthoschemes before its evaluation which per se is already a difficult task. In particular, such a procedure does not allow the characterisation of volumes of arithmetically defined or other distinguished hyperbolic 5-space forms in terms of their (number theoretical) basic data.

In this work, we describe several scissors congruence procedures in detail, providing new relations in the group $\mathcal{P}\left(\overline{\mathbb{H}^{5}}\right)$ between non-compact orthoschemes and orthoprisms. In the case of certain quasi-Coxeter polytopes (see Sect. 2.4, (11) and (12)), whose dihedral angles are related to the golden ratio, we are able to represent their volumes as explicit $\mathbb{Q}$-linear combinations of three fundamental constants, Apéry's constant $\zeta(3)$, the trilobachevsky value $\pi_{3}\left(\frac{\pi}{5}\right)$ and the volume $\mu_{5}$ of an ideal regular hyperbolic 5-simplex (see Sect. 4, Theorems I and II). This will be achieved by scissors congruence techniques and without analytic manipulations!

This approach may be of relevance by studying quantitative and arithmetic aspects of further extremal volume problems in hyperbolic 5-space. In this context, let us point out the particular role of an ideal regular hyperbolic 5-simplex $S_{\text {reg }}^{\infty}(2 \lambda)$ with dihedral angle $2 \lambda$ satisfying $\cos (2 \lambda)=1 / 4$. In fact, the volume of an arbitrary hyperbolic 5-simplex is maximal if and only if it is ideal and regular. A first analytical proof, for arbitrary dimension $n$, is due to U. Haagerup and H.J. Munkholm [6], a second and geometrical proof based on Steiner symmetrisation is due to N. Peyerimhoff [13]. This extremality property is a key, for example, in Gromov's proof [5] of Mostow rigidity.

Finally, let us mention that L. Schläfli considered the three-dimensional spherical analogues of the quasi-Coxeter polytopes in (11) and (12). By applying his reduction principle, he was able to determine their volumes and used them for the volume computation of a regular spherical simplex $S_{\text {reg }}(2 \lambda) \subset S^{3}$ as rational linear combination in terms of $\lambda \pi$ and $\pi^{2}$ (cf. [15, Sect. 30], and Sect. 4.3, Remark 5).

\section{Cycles of Orthoschemes}

\subsection{Orthoschemes and Orthoprisms}

Let $\mathbb{X}^{n}=\mathbb{S}^{n}, \mathbb{E}^{n}, \mathbb{H}^{n}$ be a standard space of constant curvature $K \in\{+1,0,-1\}$. Put $\mathbb{H}^{n}$ in the Lorentz-Minkowski vector space $\mathbb{R}^{n, 1}$ of signature $(n, 1)$ or identify it with the Klein-Beltrami model in real projective space $\mathbb{R} P^{n}$. An $n$-orthoscheme $R \subset \mathbb{X}^{n}$ is an $n$-simplex bounded by hyperplanes $H_{0}, \ldots, H_{n}$ in $\mathbb{X}^{n}$ which are such that $H_{i} \perp H_{k}$ for $|i-k|>1$. Orthoschemes can be seen as higher dimensional analogues of rightangled triangles and were introduced by L. Schläfli [15]. They are characterised by many nice metrical and orthogonality relations. Apart from right dihedral angles, an 
Fig. 1 An orthoscheme $R=p_{0} \cdots p_{5}$ in $\mathbb{X}^{5}$

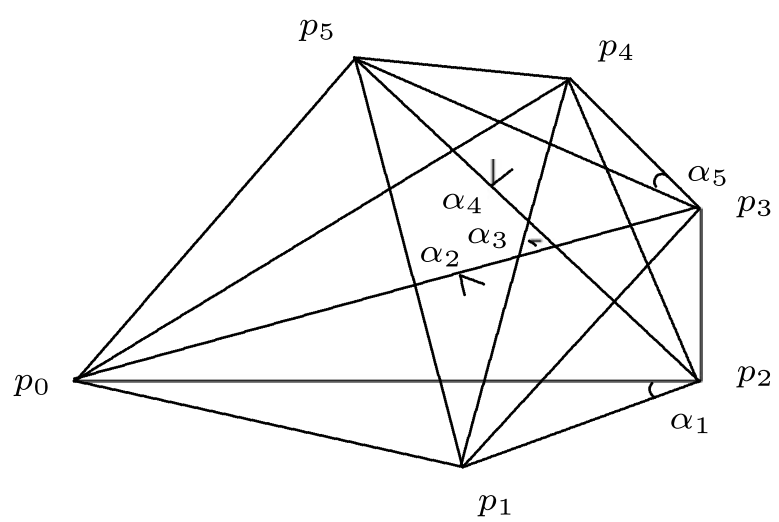

orthoscheme $R$ has dihedral angles $\alpha_{i}:=\angle\left(H_{i-1}, H_{i}\right), 1 \leq i \leq n$, which-in the hyperbolic case - are acute and form a complete system of parameters. We shall only consider orthoschemes with non-obtuse dihedral angles. For $0 \leq i \leq n$, consider the vertex $p_{i}$ opposite to the hyperplane $H_{i}$ in $R$ and write $R=p_{0} \cdots p_{n}$ (cf. Fig. 1).

The edges $p_{0} p_{1}, \ldots, p_{n-1} p_{n}$ of $R$ form an orthogonal polygonal path $\pi$ in $\mathbb{X}^{n}$ with the special property that, for $\mathbb{X}^{n}=\mathbb{H}^{n}$, at most the initial vertex $p_{0}$ and/or the final vertex $p_{n}$ of the path $\pi$ may be ideal points, i.e. points belonging to the boundary $\partial \mathbb{H}^{n}$ at infinity. In the latter case, we call $R$ simply or doubly asymptotic. By considering $\mathbb{H}^{n}$ in $\mathbb{R} P^{n}$, we may extend this process and allow the initial vertex $p_{0}$ and/or the final vertex $p_{n}$ of $\pi$ to be ultra-ideal points, that is, points lying outside the defining quadric $Q_{n, 1}=\partial \mathbb{H}^{n}$ of signature $(n, 1)$, as long as all edges of $\pi$ emanating from $p_{0}$ and/or $p_{n}$ intersect non-trivially the interior $\mathbb{H}^{n}$. We continue to call $R$ a hyperbolic $n$-orthoscheme. Notice, however, that the convex body $R \cap \mathbb{H}^{n}$ is of infinite volume. By cutting with the polar (or projectively dual) hyperplane $P_{0}$ (resp. $P_{n}$ ) associated with $p_{0}$ (resp. $p_{n}$ ) in $\mathbb{R} P^{n}$, the orthoscheme $R$ will be truncated yielding a polyhedron $R^{t}$ of finite volume in $\mathbb{H}^{n}$ with boundary formed by $H_{0}, \ldots, H_{n}$ and by the hyperplane $P_{0}$ and/or $P_{n}$ (for more details, compare [8, 9]). In the sequel, we are mainly interested in simply truncated $n$-orthoschemes or orthoprisms $R^{t}$, that is, in hyperbolic $n$-prisms with an $(n-1)$-orthoscheme basis which arise by polar truncation of $R$ in $\mathbb{R} P^{n}$ having only one ultra-ideal vertex, say $p_{0}$. By construction, the polar hyperplane $P_{0}$ has a common perpendicular with the hyperplane $H_{0}$ in $\mathbb{H}^{n}$ which coincides with the hyperbolic line defined by $p_{0}$ and $p_{1}$. We always assume that the distance between $P_{0}$ and $H_{0}$ is positive. Furthermore, since $P_{0}$ intersects orthogonally all hyperplanes through $p_{0}$ of $R$, the orthoprism $R^{t}$ has-apart from $n$ additional right dihedral angles — the same dihedral angles and parameters $\alpha_{1}, \ldots, \alpha_{n}$ as $R$, at least for $n \geq 3$. In fact, a 2-orthoprism is a Lambert quadrilateral in $\mathbb{H}^{2}$ with a single angular parameter $\alpha_{1} \in\left[0, \frac{\pi}{2}[\right.$.

\subsection{Graphs of Orthoschemes and Orthoprisms}

Let $R$ be an $n$-orthoscheme in $\mathbb{X}^{n}$ with vertices $p_{0}, \ldots, p_{n}$ lying opposite to the bounding hyperplanes $H_{0}, \ldots, H_{n}$, and denote by $\alpha_{i}=\angle\left(H_{i-1}, H_{i}\right)$ the dihedral angle formed by $H_{i-1}, H_{i}$. If $p_{0}$ is ultra-ideal, denote by $R^{t}$ the orthoprism given by the truncation of $R$ by means of the polar hyperplane $P_{0}$. 
By discarding the hyperplane $H_{0}$ from the boundary of $R$, the configuration $H_{1}, \ldots, H_{n}$ yields a simplicial cone $R_{p_{0}}$ with apex $p_{0}$. If $p_{0}$ is an ordinary point in $\mathbb{X}^{n}$ (resp. an ideal point of $\mathbb{H}^{n}$ ), then the apex geometry of $R_{p_{0}}$ in $\mathbb{X}^{n}$ is given by a spherical (resp. Euclidean) $(n-1)$-orthoscheme with dihedral angles $\alpha_{2}, \ldots, \alpha_{n}$. If $p_{0}$ is an ultra-ideal point in $\mathbb{R} P^{n}$, the apex geometry in $\mathbb{H}^{n}$ of the cone $R_{p_{0}}$ with apex $p_{0}$ in $\mathbb{R} P^{n}$ is given by a hyperbolic $(n-1)$-orthoscheme with dihedral angles $\alpha_{2}, \ldots, \alpha_{n}$. The latter is identical with the prism basis of $R^{t}$.

Let us associate with an $n$-orthoscheme $R$ (and $R^{t}$ ) a graph $\Sigma=\Sigma(R)$, characterising $R$ up to congruence, by assigning to each hyperplane $H_{i}$ a node $v_{i}$ in $\Sigma$. Two nodes are joined by an edge if the corresponding hyperplanes are not orthogonal. We attach the weight $\alpha_{i}$ to the edge joining the nodes of the consecutive hyperplanes if they intersect under the dihedral angle $\alpha_{i}$. A subgraph of a graph $\Sigma=\Sigma(R)$ arises by discarding nodes together with their edges. Observe that $\Sigma$ consists of (at most $n+1)$ connected components of linear graphs. In the hyperbolic finite volume case, it is easy to see that $\Sigma$ is always connected.

To every graph $\Sigma(R)$ as above corresponds the (symmetric) Gram matrix $G(R)=$ $\left(g_{i k}\right)$ of $R$ which is of order $n+1$, with $g_{i i}=1, g_{i, i+1}=-\cos \alpha_{i+1} \leq 0$ and $g_{i k}=0$ otherwise $(0 \leq i<k \leq n)$. On the other hand, if $G=\left(g_{i k}\right)$ is an indecomposable symmetric matrix of order $n+1$ with $g_{i i}=1, g_{i, i+1} \leq 0$ and $g_{i k}=0$ otherwise, then $G$ can be realised as Gram matrix $G(R)$ for a $n$-orthoscheme $R$ in $\mathbb{X}^{n}$ as follows (cf. [16, Sect. 2] and [14, Sects. 7.2, 7.3]). Denote by $G_{l}$ the principal submatrix of order $n$ of $G$ arising by removing the $(l+1)$-th row and the $(l+1)$-th column.

(1) If $G$ is positive definite, then $G=G(R)$ for a spherical $n$-orthoscheme $R$ defined up to isometry. Both $G(R)$ and the graph $\Sigma$ are called elliptic.

(2) If $G$ is positive semidefinite, then $G=G(R)$ for a Euclidean $n$-orthoscheme $R$ defined up to similarity. Both, $G(R)$ and the graph $\Sigma$ are called parabolic.

(3) If $G$ is of signature $(n, 1)$, then $G=G(R)$ for a hyperbolic $n$-orthoscheme $R$ (of finite or infinite volume) defined up to isometry. The matrix $G(R)$ and the graph $\Sigma(R)$ are called hyperbolic. We distinguish further the following subcases.

(a) If all principal submatrices of $G$ are elliptic, then $R$ is a compact orthoscheme. Its graph $\Sigma=\Sigma(R)$ will be drawn according to

$$
\Sigma: \circ \stackrel{\alpha_{1}}{\circ} \circ-\cdots-\circ \stackrel{\alpha_{n}}{\circ}
$$

(b) If, apart from elliptic principal submatrices of $G$, only $G_{0}$ (and $G_{n}$ ) is parabolic, then $R$ is a simply (doubly) asymptotic orthoscheme. In particular, if $R$ is simply (doubly) asymptotic of finite volume with $G_{0}$ (and $G_{n}$ ) parabolic, we blacken the node $v_{0}$ (and $v_{n}$ ) and write

$$
\Sigma: \bullet \frac{\alpha_{1}}{-} \circ-\cdots-\circ \frac{\alpha_{n}}{\circ} \text {. }
$$

(c) If, among all principal submatrices of $G$, precisely $G_{0}$ is hyperbolic, then $R$ is an orthoscheme with ultra-ideal vertex $p_{0}$ and associated orthoprism $R^{t}$ of finite volume. The graph $\Sigma=\Sigma\left(R^{t}\right)$ of $R^{t}$ is given by the graph $\Sigma(R)$ by putting a diamond in place of the node $\nu_{0}$ (indicating that $P_{0}$ is added to the 
hyperplane configuration $H_{0}, \ldots, H_{n}$ bounding $R$ ). A compact $n$-orthoprism $R^{t}$ is therefore described by

$$
\Sigma: \diamond \frac{\alpha_{1}}{2} \circ-\cdots-\circ \frac{\alpha_{n}}{\circ} \circ
$$

\subsection{The Coxeter and Pseudo-Coxeter Cases}

Consider a convex $n$-polyhedron $P$ in $\mathbb{X}^{n}$. If all its dihedral angles are of the form $\pi / k$ with $k \geq 2$, the polyhedron $P$ is a Coxeter polyhedron. In the theory of regular polyhedra and regular honeycombs, the family of Coxeter orthoschemes plays an important role. In fact, Coxeter orthoschemes provide fundamental domains for the associated symmetry groups. They were classified by Coxeter, Lannér and KoszulChein (cf. [16] for lists and references). The family of simply and doubly truncated Coxeter orthoschemes in $\mathbb{H}^{n}$ was studied and classified by H.-C. Im Hof (cf. [8]). It is noteworthy that hyperbolic Coxeter orthoschemes, truncated or not, exist only up to dimension 9. In the compact case, they exist only up to dimension 5.

A convex $n$-polyhedron $P \subset \mathbb{X}^{n}$ is called a pseudo-Coxeter polyhedron if all its dihedral angles are commensurable with $\pi$. They arise naturally in the study of regular star-polyhedra and star-honeycombs (cf. [2, p. $161 \mathrm{ff}]$ ). The notion of graph of a pseudo-Coxeter orthoscheme is defined in an analogous way by a graph with edge weigths $\frac{q}{p}$ corresponding to (non-right) dihedral angles of the form $p \pi / q$ for integers $p \geq 1, q \geq 3$. Of interest will be, among others, the pseudo-Coxeter orthoschemes in $\mathbb{H}^{5}$ given by

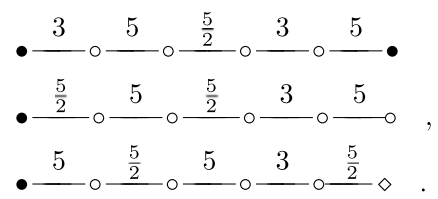

In the sequel, by considering dissections of certain Coxeter and quasi-Coxeter polyhedra, new polyhedra will arise with dihedral angles of mixed type, that is, some angles are commensurable with $\pi$, some are not. In order to keep the notations as concise as possible, we describe these polyhedra by graphs with rational weights in the first case and with letters such as $\alpha, \lambda, \omega$ representing angular parameters in the second case (see the examples (5), (6) below).

Remark Observe that $\cos ^{2} \frac{\pi}{3}+\cos ^{2} \frac{\pi}{5}+\cos ^{2} \frac{2 \pi}{5}=1$ which is responsible for the parabolicity of the subgraphs in (1). In general, the parabolicity of a graph

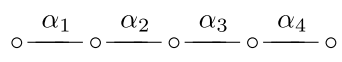

is equivalent to the condition that (cf. [2, p. 264])

$$
\frac{\cos ^{2} \alpha_{2}}{\sin ^{2} \alpha_{1}}+\frac{\cos ^{2} \alpha_{3}}{\sin ^{2} \alpha_{4}}=1 .
$$




\subsection{Orthoscheme Cycles}

Consider a doubly asymptotic 5-orthoscheme $R$ with graph

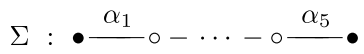

and define the angle $\left.\alpha_{0} \in\right] 0, \frac{\pi}{2}[$ such that the graph

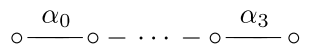

is parabolic according to (2). Then, by [10, Sect. 1.4, Lemma], the graphs

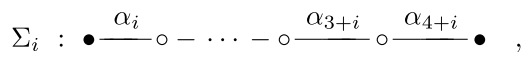

wherein indices $i \geq 1$ are taken modulo 6 , form a $\Sigma$-cycle of length 6 consisting of six different doubly asymptotic 5-orthoschemes where two neighbours share a vertex at infinity. According to the weights, a cycle may consist of tuples of isometric members and "collapse" to a cycle consisting of a smaller number of non-isometric members. This phenomenon reduces the length of the cycle, accordingly. Here are the most prominent examples for the subsequent investigations:

$$
\begin{aligned}
& U_{i} \quad: \quad \stackrel{\frac{5}{2}}{\circ} \stackrel{5}{\longrightarrow} \circ \stackrel{\frac{5}{2}}{\circ} \stackrel{5}{ } \circ \stackrel{\frac{5}{2}}{\bullet}, \quad i \bmod .2 ; \\
& V_{i}: \bullet \frac{3}{-} \circ \frac{5}{\circ} \circ \stackrel{\frac{5}{2}}{\circ} \stackrel{3}{\longrightarrow} \circ \frac{5}{-}, \quad, \quad i \bmod .3 ; \\
& W_{i} \quad: \quad \stackrel{3}{\square} \circ \frac{3}{-} \circ \stackrel{\frac{5}{2}}{\circ} \stackrel{\omega_{1}}{\longleftarrow} \stackrel{\omega_{2}}{ } \bullet, \quad i \bmod .6 \\
& Z_{i}: \bullet \stackrel{\lambda}{-} \circ \stackrel{3}{-} \circ \stackrel{3}{\longrightarrow} \circ \stackrel{3}{-} \stackrel{\lambda}{\longrightarrow}, \quad i \bmod .4,
\end{aligned}
$$

where the weights in (5) and (6) are given by the conditions

$$
\begin{gathered}
0<\lambda, \omega_{1}, \omega_{2}<\frac{\pi}{2} \quad \text { with } \\
\cos (2 \lambda)=\frac{1}{4}, \quad \omega_{1}=\frac{\pi}{3}-\lambda, \quad \omega_{2}=\frac{\pi}{3}+\omega_{1} .
\end{gathered}
$$

Denote by $\tau$ the golden ratio given by $\tau=2 \cos \frac{\pi}{5}=\frac{1+\sqrt{5}}{2}$ satisfying the quadratic equation $\tau^{2}=\tau+1$. Then, we deduce the following relations:

$$
\sin \omega_{1}=\sqrt{\frac{3}{8}}(\tau-1), \quad \sin \omega_{2}=\sqrt{\frac{3}{8}} \tau .
$$

Observe that the $V$-cycle (4) is a realisation of the cycle of length 3 given by

$$
\Sigma_{i}: \bullet \frac{\alpha}{\circ} \circ \stackrel{\beta}{\gamma} \circ \frac{\alpha}{\circ} \circ \stackrel{\beta}{\longrightarrow}, \quad i \bmod .3,
$$


where $0<\alpha, \beta, \gamma<\frac{\pi}{2}$ satisfy the equation (cf. also (1))

$$
\cos ^{2} \alpha+\cos ^{2} \beta+\cos ^{2} \gamma=1 \text {. }
$$

The $Z$-cycle (6) of length 4 is not of type (9), (10). However, its members belong to different dissections of an ideal regular 5-simplex $S_{\text {reg }}^{\infty}(2 \lambda)$ with dihedral angle $2 \lambda$ satisfying (7) (see Sect. 3.2, Remark 2).

Closely related to the doubly asymptotic 5-orthoschemes above is the following group of simply asymptotic 5-orthoschemes:

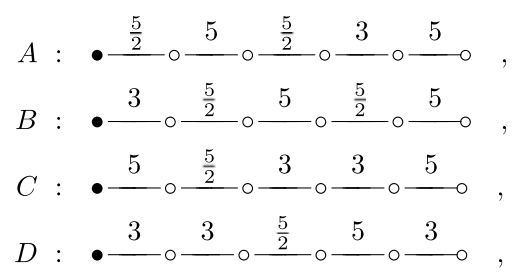

as well as the following group of (simply) asymptotic 5-orthoprisms:

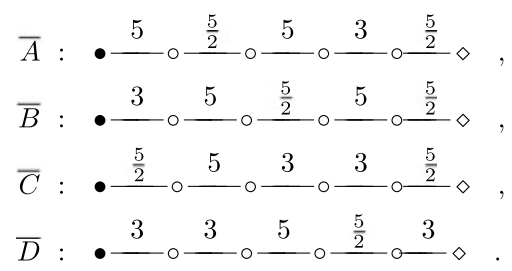

We shall be able to determine all volumes for (11) and (12) as rational linear combinations of the characteristic units $\zeta(3), \Omega_{3}\left(\frac{\pi}{5}\right)$. Then, it will be possible to relate them to some members of (5) and to some members of the $Z$-cycle and therefore to the volume $\mu_{5}:=\operatorname{vol}_{5}\left(S_{\text {reg }}(2 \lambda)\right)$ in hyperbolic 5-space (cf. Sect. 4).

\section{Scissors Congruences in Hyperbolic 5-Space}

In the hyperbolic space, asymptotic orthoschemes play a particularly important role in the context of scissors congruence.

Let $Y^{n}$ be either $\mathbb{H}^{n}$ or $\overline{\mathbb{H}^{n}}=\mathbb{H}^{n} \cup \partial \mathbb{H}^{n}$. Recall that the scissors congruence group $\mathcal{P}\left(Y^{n}\right)$ is the abelian group generated by $[P]$ for each polyhedron $P$ in $Y^{n}$ equipped with the relations (i) $[P \sqcup Q]=[P]+[Q]$ ( $\sqcup$ denotes disjoint interior union) and (ii) $[P]=[Q]$ for $P$ isometric to $Q$. For $n \geq 2$, the group $\mathcal{P}\left(\mathbb{H}^{n}\right)$ is generated by the classes of simply asymptotic orthoschemes, while, for $n>1$ odd, the group $\mathcal{P}\left(\overline{\mathbb{H}^{n}}\right)$ is generated by the classes of doubly asymptotic orthoschemes (for references, see for example [3]). The proof of these results is based on several cutting and pasting procedures for orthoschemes (cf. Sects. 3.2 and Sect. 3.3 below). By applying the homomorphism given by the volume functional, these two scissors congruences, combined with some more relations including the barycentric dissection for regular simplices, will enable us to derive the volumes of all polytopes in (11) and (12) and 
Fig. 2 Bisecting the angle of parallelism

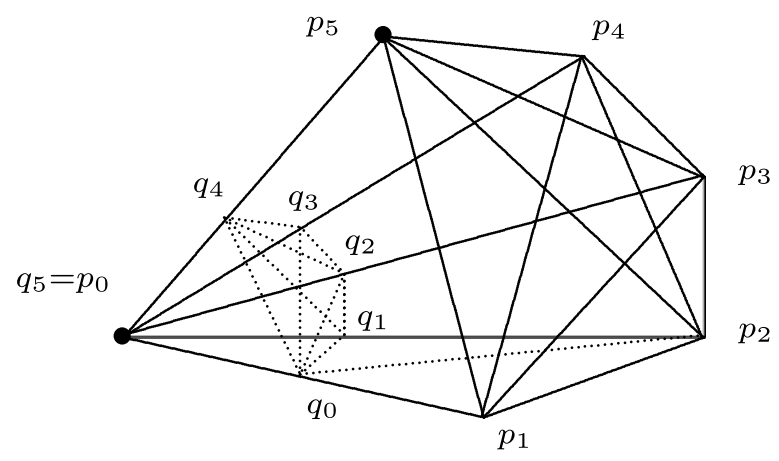

to relate the volume of some members of the $W$-cycle to the volume $\mu_{5}$ of an ideal regular 5-simplex. This will be achieved by elegant decomposition relations, avoiding hard analytic computations. In the sequel, we present the scissors congruences in detail for asymptotic orthoschemes and orthoprisms of dimension $n=5$, only.

\subsection{Bisecting the Angle of Parallelism}

Let $R=p_{0} \cdots p_{5}$ denote a doubly asymptotic 5-orthoscheme with vertices $p_{0}, \ldots, p_{5}$ and with graph

$$
\Sigma(R) \quad: \quad \bullet \frac{\alpha_{1}}{-} \circ \frac{\alpha_{2}}{{ }^{\prime}} \circ \frac{\alpha_{3}}{2} \circ \frac{\alpha_{4}}{2} \circ \frac{\alpha_{5}}{\bullet}
$$

The dihedral angles $\alpha_{1}$ and $\alpha_{5}$ appear as angles of parallelism in simply asymptotic triangles. For instance, $\alpha_{1}$ is the angle of parallelism in the triangle $p_{0} p_{1} p_{2}$ (cf. Fig. 1) whence

$$
\sin \alpha_{1}=\frac{1}{\cosh p_{1} p_{2}} .
$$

Write $\alpha_{1}=: 2 \alpha$. In the sequel, we decompose $R$ into 6 simply asymptotic orthoschemes two of which are isometric to one another with angle of parallelism equal to $\alpha$. Draw the angle bisector $l$ of $\alpha_{1}$ in the triangle $p_{0} p_{1} p_{2}$ and denote by $q_{0}:=l \cap p_{0} p_{1}$ the intersection of $l$ with the line defined by $p_{0}, p_{1}$. Consider the hyperplane $H$ through $q_{0}$ which is orthogonal to the line $p_{0} p_{5}$ and define $q_{i}:=H \cap p_{0} p_{i+1}$ for $1 \leq i \leq 4$ (cf. Fig. 2).

Write $q_{5}:=p_{0} \in \partial \mathbb{H}^{5}$ and form the convex hull

$$
R_{k}:=q_{0} \cdots q_{k-1} p_{k} p_{k+1} \cdots p_{5}, \quad 0 \leq k \leq 6
$$

By construction, each $R_{k}, 0 \leq k \leq 6$, is a 5-orthoscheme with $R=R_{0}$ and $R_{6}=$ : $Q=q_{0} \cdots q_{5}$. For $k \neq 0$, the orthoscheme $R_{k}$ is simply asymptotic. Furthermore, $R_{1}$ is isometric to $R_{2}$, and $R_{5}$ is isometric to $R_{6}$ (cf. Fig. 1 and use angle parallelism). The construction (14) yields the scissors congruence relation

$$
[R]=\sum_{k=1}^{6}\left[R_{k}\right]=2\left[R_{2}\right]+\left[R_{3}\right]+\left[R_{4}\right]+2\left[R_{5}\right]
$$


called the bisection of the angle of parallelism. In the following, we determine the metric properties of the dissecting orthoschemes. Denote the graphs associated with $R_{2}, \ldots, R_{5}$ by

$$
\begin{aligned}
& \Sigma\left(R_{2}\right) \quad: \quad \circ \stackrel{\alpha}{-} \circ \stackrel{u}{-} \circ \frac{\alpha_{3}}{2} \circ \frac{\alpha_{4}}{2} \circ \stackrel{\alpha_{5}}{-} \\
& \Sigma\left(R_{3}\right) \quad: \quad \circ \frac{\alpha_{2}}{-\bar{u}} \circ \frac{v}{-} \circ \frac{x}{-} \circ \frac{\alpha_{5}}{\bullet} \\
& \Sigma\left(R_{4}\right) \quad: \quad \circ \frac{\alpha_{2}}{\circ} \circ \frac{\alpha_{3}}{\alpha} \circ \frac{w}{-} \circ \stackrel{\bar{z}}{-} \\
& \Sigma\left(R_{5}\right): \text { : } \frac{\alpha_{2}}{-} \circ \frac{\alpha_{3}}{-} \circ \frac{\alpha_{4}}{-} \circ \frac{\alpha_{5}}{-} \circ \stackrel{z}{-} \text {, }
\end{aligned}
$$

where we put $\bar{\omega}:=\pi-2 \omega$. By (2), the parabolicity of the subgraph of $R_{2}$ associated with the common vertex $p_{5}$ yields

$$
\cos ^{2} u=\left(1-\frac{\cos ^{2} \alpha_{3}}{\sin ^{2} \alpha_{4}}\right) \sin ^{2} \alpha .
$$

In order to determine the remaining parameters in (16), we consider the 3 -face $q_{0} q_{1} q_{2} q_{3}$ in $Q$ (cf. Fig. 2). The vertex figure associated with $q_{3}$ is a right-angled spherical triangle with angles $\alpha_{2}, \alpha_{3}$ so that its edge length $\delta$ opposite to $\alpha_{3}$ appears as the planar angle opposite to the edge $q_{1} q_{2}$ in the face $q_{1} q_{2} q_{3}$. By (13) and with some non-Euclidean trigonometry, one easily computes $\delta$ and the lengths of the orthogonal edge path in $q_{0} q_{1} q_{2} q_{3}$ according to

$$
\begin{gathered}
\cosh q_{0} q_{1}=\frac{2 \cos ^{2} \alpha}{\sqrt{4 \cos ^{2} \alpha-1}}, \quad \cos \delta=\frac{\cos \alpha_{3}}{\sin \alpha_{2}}, \\
\sinh q_{1} q_{2}=\tanh q_{0} q_{1} \cdot \cot \alpha_{2}, \quad \sinh q_{2} q_{3}=\tanh q_{1} q_{2} \cdot \cot \delta .
\end{gathered}
$$

In a similar way, by considering the vertex figures associated with $q_{2}$ in the 3-faces $q_{2} q_{3} q_{4} p_{5}$ of $R_{5}, q_{2} q_{3} p_{4} p_{5}$ of $R_{4}$ and $q_{2} p_{3} p_{4} p_{5}$ of $R_{3}$ respectively, we obtain the relations

$$
\tanh q_{2} q_{3}=\cot z \cot \alpha_{5}=\cot y \cot \bar{z} ; \quad \sin x=\frac{\cos \alpha_{5} \sin y}{\cos \bar{z}} .
$$

The remaining parameters $v, w$ are determined by a parabolicity condition (2).

Example 1 For the pseudo-Coxeter orthoscheme $R$ with graph given by (cf. (3))

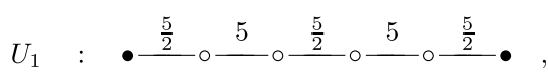

one derives the relations

$$
\begin{aligned}
& {\left[R_{2}\right]=\left[R_{3}\right]=\left[\circ \frac{5}{5} \circ \frac{3}{\circ} \circ \frac{\frac{5}{2}}{\circ} \circ \frac{5}{\circ} \circ \frac{\frac{5}{2}}{\bullet}\right]=[A],} \\
& {\left[R_{4}\right]=\left[R_{5}\right]=\left[\circ \frac{5}{2} \circ \circ \div \frac{5}{2} \circ \div\right]=[B] .}
\end{aligned}
$$

Hence, by (15), it follows that

$$
\left[U_{1}\right]=3[A]+3[B] .
$$


Example 2 For the pseudo-Coxeter orthoscheme $R$ with graph (cf. (4))

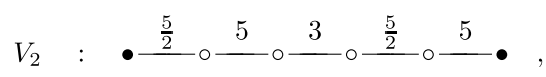

one derives the relations

$$
\begin{aligned}
& {\left[R_{2}\right]=\left[R_{3}\right]=\left[R_{4}\right]=\left[\circ \stackrel{5}{\circ} \circ \stackrel{3}{5} \circ \frac{3}{3} \circ \stackrel{\frac{5}{2}}{\circ} \stackrel{5}{-\bullet}\right]=[C],} \\
& {\left[R_{5}\right]=\left[\circ \stackrel{5}{\circ} \circ \stackrel{3}{\circ} \stackrel{\frac{5}{2}}{\circ} \stackrel{5}{\circ} \stackrel{\frac{5}{2}}{\bullet}\right]=[A]}
\end{aligned}
$$

Again, by (15), we obtain that

$$
\left[V_{2}\right]=2[A]+4[C] .
$$

Example 3 For the cycle neighbour $R$ of $V_{2}$ in (4) given by

$$
V_{3}: \bullet \frac{\frac{5}{2}}{\circ} \circ \frac{3}{\circ} \circ \stackrel{5}{\circ} \stackrel{\frac{5}{2}}{\circ} \stackrel{3}{\longrightarrow},
$$

one derives the relations

$$
\begin{aligned}
{\left[R_{2}\right] } & =\left[\circ \frac{5}{3} \circ \frac{\frac{5}{2}}{5} \frac{5}{\circ} \circ \frac{\frac{5}{2}}{\circ} \frac{3}{3} \bullet\right]=[B], \\
{\left[R_{3}\right]=\left[R_{4}\right]=\left[R_{5}\right] } & =\left[\circ \stackrel{3}{\circ} \stackrel{5}{\frac{5}{2}} \circ \frac{3}{\circ} \circ\right]=[D] .
\end{aligned}
$$

Here, we obtain that

$$
\left[V_{3}\right]=2[B]+4[D]
$$

Remark 1 It is not hard to generalise the dissection (15) by dissecting the angle of parallelism $\alpha_{1}=\beta_{1}+\beta_{2}$ for arbitrary $0<\beta_{1}, \beta_{2}<\alpha_{1}$.

\subsection{Reversing to Infinity}

Let $R=p_{0} \cdots p_{5}$ be a simply asymptotic 5-orthoscheme with vertices $p_{0}, \ldots, p_{4} \in$ $\mathbb{H}^{5}$ and with $p_{5} \in \partial \mathbb{H}^{5}$ encoded by the graph

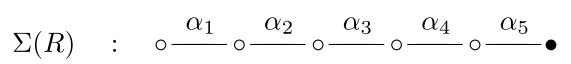

Prolong the (finite) oriented geodesic segment $p_{0} p_{1}$ from $p_{0}$ to $p_{1}$ in the reversed sense to a geodesic half-line $l_{1}$ with limit point $q_{0} \in \partial \mathbb{H}^{5}$, say. In the same way, prolong all oriented edges $p_{0} p_{i}$ in the reversed sense in order to obtain geodesic half-lines $l_{i}$ for $2 \leq i \leq 5$ (cf. Fig. 3). Consider the hyperplane $H$ through $q_{0}$ which is orthogonal to $l_{5}$ and define the points $q_{i}:=H \cap l_{i+1}$ for $i=1, \ldots, 4$, and let $q_{5}:=p_{0}$. Form the convex hull

$$
R_{k}:=q_{0} \cdots q_{k-1} p_{k} \cdots p_{5}, \quad 0 \leq k \leq 6
$$




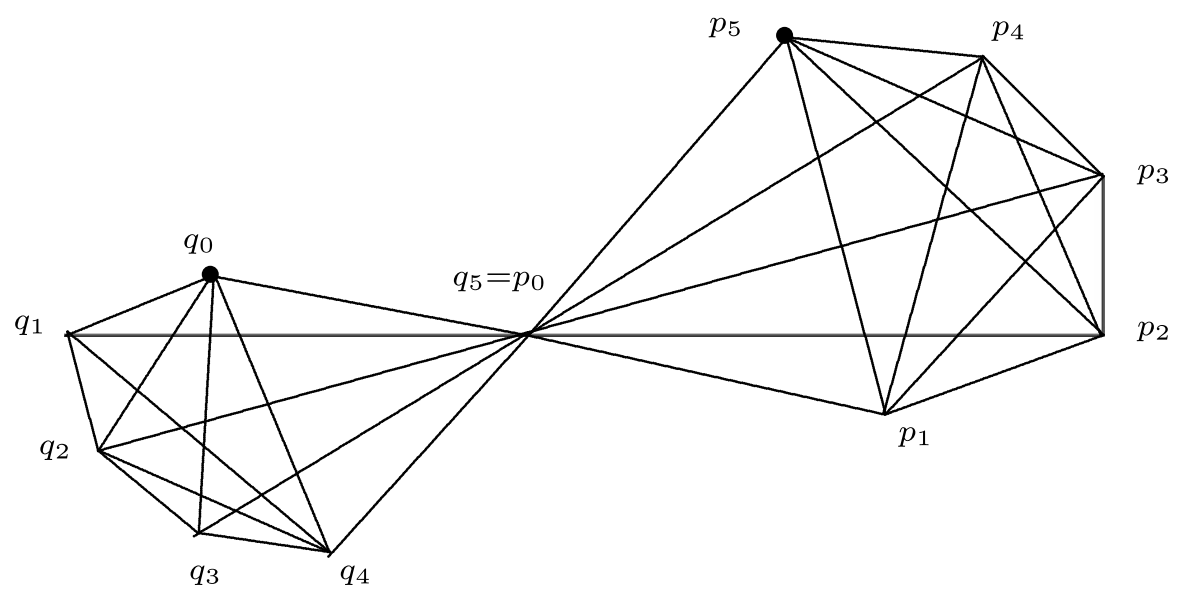

Fig. 3 Reversing to infinity

It follows easily (cf. also [3, Theorem (2.6), (ii)]) that $R_{0}=R$ and $R_{6}=: Q$ are simply asymptotic and that $R_{k}, 1 \leq k \leq 5$, are doubly asymptotic orthoschemes satisfying the relation

$$
[R]+[Q]=\sum_{k=1}^{5}(-1)^{k-1}\left[R_{k}\right] .
$$

Since $Q$ is simply asymptotic with vertex $q_{0} \in \partial \mathbb{H}^{5}$ and with spherical vertex figure at $q_{5}=p_{0}$ isometric to that one of $R$ at $p_{0}$, we deduce that its graph equals

$$
\Sigma(Q) \quad: \quad \bullet \frac{\alpha_{2}}{-} \circ \frac{\alpha_{3}}{2} \circ \frac{\alpha_{4}}{\circ} \circ \frac{\alpha_{5}}{2} \circ \frac{\alpha_{6}}{\circ} \circ
$$

where $\left.\alpha_{6} \in\right] 0, \frac{\pi}{2}[$ is given by the parabolicity condition (2), that is,

$$
\frac{\cos ^{2} \alpha_{4}}{\sin ^{2} \alpha_{3}}+\frac{\cos ^{2} \alpha_{5}}{\sin ^{2} \alpha_{6}}=1 .
$$

For the graphs of the orthoschemes $R_{k}, 1 \leq k \leq 5$, write (cf. also Fig. 1)

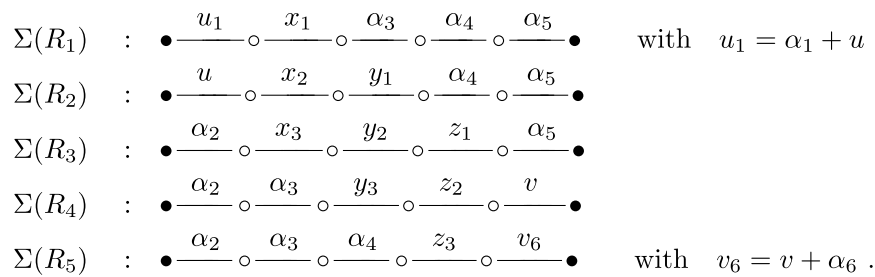

The dihedral angles $x_{1}, u_{1}, z_{3}, v_{6}$ (and therefore $u, v$ ) can be determined by the parabolicity condition (2). Then, the dihedral angles $x_{2}, z_{2}$ follow from the conditions

$$
\tan u \tan x_{2}=\tan \alpha_{4} \tan \alpha_{5}, \quad \tan \alpha_{2} \tan \alpha_{3}=\tan z_{2} \tan v,
$$


Fig. 4 Vertex figures at $q_{0}$

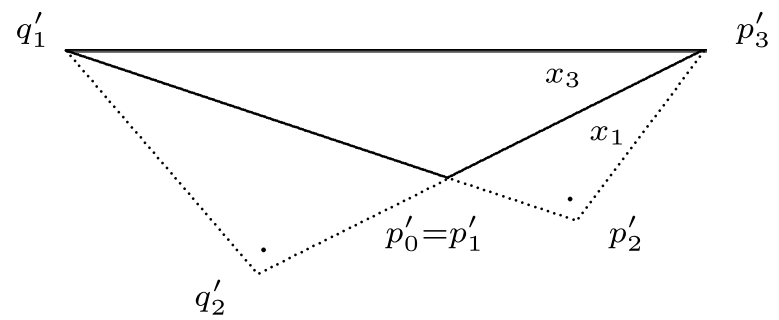

which reflect the bi-asymptoticity of the 5-orthoschemes $R_{2}$ and $R_{4}$ (cf. [10, Sect. 1.4, Lemma]). Again, the parameters $y_{1}, y_{3}$ follow from (2). As for $x_{3}, z_{1}$, observe that

$$
x_{1}+x_{3}=x_{2}, \quad z_{1}+z_{3}=z_{2} .
$$

Indeed, for the first part of (27), consider the 3-faces $q_{0} p_{1} p_{2} p_{3}$ of $R_{1}, q_{0} q_{1} p_{2} p_{3}$ of $R_{2}$ and $q_{0} q_{1} q_{2} q_{3}$ of $R_{3}$, respectively. The (Euclidean) vertex figures associated with $q_{0}$ yield a picture (with obvious labellings) as drawn in Fig. 4. Now, the first relation in (27) is the angle sum $x_{2}=x_{1}+x_{3}$ at $p_{3}^{\prime}$. The second relation of (27) is verified accordingly by considering the vertex $p_{5}$. Finally, the dihedral angle $y_{2}$ follows from the parabolicity condition (2).

Example 1 For the pseudo-Coxeter orthoscheme $R$ with graph (cf. (11))

$$
B=\Sigma(R) \quad: \quad \circ \frac{5}{-\frac{5}{2}} \circ \frac{5}{-} \circ \stackrel{\frac{5}{2}}{\circ} \stackrel{3}{\longrightarrow}
$$

one obtains (cf. Sect. 2.3, Remark, and (3)-(5), (7))

$$
\begin{aligned}
& A=\Sigma(Q) \quad: \quad \bullet \frac{\frac{5}{2}}{2} \circ \frac{5}{-} \circ \stackrel{\frac{5}{2}}{\longrightarrow} \circ \frac{5}{5} \circ \\
& V_{3}=\Sigma\left(R_{1}\right) \quad: \quad \bullet \frac{\frac{5}{2}}{5} \circ \frac{3}{5} \circ \stackrel{\frac{5}{2}}{\circ} \stackrel{3}{\longrightarrow} \\
& W_{2}=\Sigma\left(R_{2}\right) \quad: \quad \bullet \frac{5}{\circ} \circ \stackrel{\omega_{2}}{\longrightarrow} \circ \omega_{1} \circ \stackrel{\frac{5}{2}}{-} \circ \frac{3}{-} \text {. } \\
& W_{3}=\Sigma\left(R_{3}\right) \quad: \quad \bullet \frac{\frac{5}{2}}{\circ} \circ \frac{\omega_{1}}{5} \circ \frac{\omega_{2}}{-} \circ \frac{5}{-} \circ \\
& V_{2}=\Sigma\left(R_{4}\right) \quad: \quad \bullet \frac{\frac{5}{2}}{\circ} \stackrel{5}{\longrightarrow} \circ \stackrel{3}{\longleftarrow} \stackrel{\frac{5}{2}}{\longleftarrow} \stackrel{5}{\longrightarrow} \\
& U_{1}=\Sigma\left(R_{5}\right) \quad: \quad \bullet \frac{\frac{5}{2}}{\circ} \stackrel{5}{\longrightarrow} \stackrel{\frac{5}{2}}{\circ} \stackrel{5}{\longrightarrow} \stackrel{\frac{5}{2}}{\bullet}
\end{aligned}
$$

Hence, by (23), we deduce that

$$
[A]+[B]=\left[W_{3}\right]-\left[W_{2}\right]+\left[U_{1}\right]+\left[V_{3}\right]-\left[V_{2}\right] .
$$

Notice that we obtain the identical relation (29) by starting with an orthoscheme $R$ given by the graph $\Sigma(R)=A$.

Example 2 For the pseudo-Coxeter orthoscheme $R$ with graph (cf. Sect. 3.1)

$$
D=\Sigma(R) \quad: \quad \circ \stackrel{3}{\longrightarrow} \circ \stackrel{5}{-} \circ \stackrel{\frac{5}{2}}{\longrightarrow} \stackrel{3}{\longrightarrow} \stackrel{3}{\longrightarrow},
$$


one obtains (cf. Sect. 2.3, Remark, and (3)-(5), (7))

$$
\begin{aligned}
& C=\Sigma(Q) \quad: \quad \bullet \frac{5}{2} \circ \stackrel{\frac{5}{2}}{\longrightarrow} \stackrel{3}{\longrightarrow} \circ \stackrel{5}{\longrightarrow} \circ \\
& W_{1}=\Sigma\left(R_{1}\right) \quad: \quad \bullet \frac{\omega_{2}}{\circ} \circ \stackrel{\omega_{1}}{\circ} \stackrel{\frac{5}{2}}{\circ} \stackrel{3}{-} \circ \frac{3}{\omega_{1}} \bullet \\
& W_{4}=\Sigma\left(R_{2}\right) \quad: \quad \bullet \frac{\omega_{1}}{5} \circ \frac{\omega_{2}}{-} \circ \frac{5}{-} \circ \stackrel{3}{-} \text {. } \\
& V_{1}=\Sigma\left(R_{3}\right) \quad: \quad \bullet \frac{5}{5} \circ \stackrel{3}{\frac{\frac{5}{2}}{5} \circ} \stackrel{5}{-} \stackrel{3}{\longrightarrow} \bullet
\end{aligned}
$$

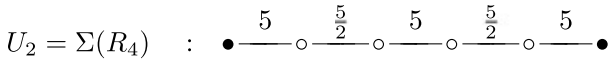

$$
\begin{aligned}
& V_{2}=\Sigma\left(R_{5}\right) \quad: \quad \bullet \frac{5}{\circ} \circ \stackrel{\frac{5}{2}}{3} \circ \frac{5}{\circ} \stackrel{\frac{5}{2}}{\bullet}
\end{aligned}
$$

Hence, by (23), we deduce that

$$
[C]+[D]=\left[W_{1}\right]-\left[W_{4}\right]-\left[U_{2}\right]+\left[V_{1}\right]+\left[V_{2}\right] .
$$

Observe that the relations obtained so far do not allow as yet to express the elements $[A],[B],[C],[D]$ only in terms of members of the $U$ - and the $V$-cycle (3) and (4) whose volumes are well known (cf. Sect. 4).

Remark 2 Consider the orthoscheme $R$ with graph

$$
Z=\Sigma(R) \quad: \quad \circ \stackrel{\lambda}{\longrightarrow} \circ \stackrel{3}{\longrightarrow} \circ \stackrel{3}{\longrightarrow} \circ \stackrel{3}{\longrightarrow}
$$

which is related to the orthoscheme cycle (6) associated with an ideal regular hyperbolic 5-simplex $S:=S_{\text {reg }}^{\infty}(2 \lambda)$ of dihedral angle $2 \lambda$ given by (7). By reversing to infinity according to (23)-(27), we deduce easily the scissors relation

$$
\begin{aligned}
& 2[Z]=2\left[Z_{2}\right]-2\left[Z_{3}\right]+\left[Z_{4}\right]=
\end{aligned}
$$

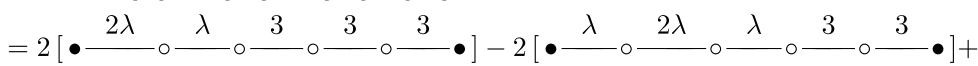

$$
\begin{aligned}
& +[\bullet-3 \stackrel{\lambda}{-} \circ \stackrel{2 \lambda}{-} \circ \stackrel{\lambda}{-} \stackrel{3}{-} \bullet] \text {, }
\end{aligned}
$$

which is compatible with the well-known barycentric subdivison for $S:=S_{\text {reg }}^{\infty}(2 \lambda)$. More precisely, let $c$ be the centre of gravity and $q$ one of the vertices at infinity of $S$. By drawing successively perpendiculars starting from $c$ or from $q$, or by applying the dissection procedure above and in Sect. 3.3 below (cf. [3, (7.4), p. 147], for example), one obtains the scissors relations

$$
\begin{aligned}
{[S] } & =720\left[\circ \frac{\lambda}{2 \lambda} \circ \frac{3}{\lambda} \circ \frac{3}{\circ} \circ \frac{3}{\circ} \circ \frac{3}{\bullet} \bullet\right] \\
& =120\left[\bullet \frac{3}{\lambda} \circ \frac{3}{2 \lambda} \bullet \cdot\right]=120\left[Z_{2}\right] \\
& =48\left[\bullet \frac{\lambda}{2} \circ \frac{\lambda}{\lambda} \circ \frac{3}{2 \lambda} \circ \frac{3}{\lambda} \bullet\right]=48\left[Z_{3}\right] \\
& =36\left[\bullet \frac{3}{\circ} \circ \frac{3}{\bullet} \bullet \cdot\right]=36\left[Z_{4}\right]
\end{aligned}
$$


Fig. 5 Extending to infinity

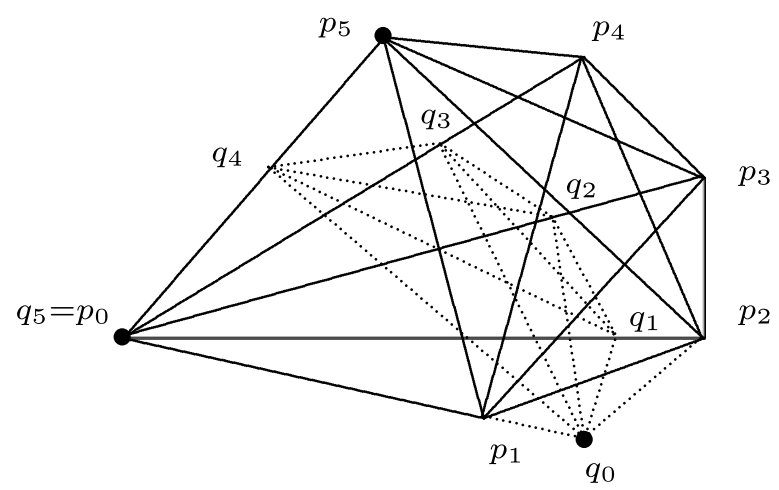

In particular, we deduce that

$$
\begin{aligned}
\mu_{5}:=\operatorname{vol}_{5}\left(S_{\mathrm{reg}}^{\infty}(2 \lambda)\right)=\operatorname{vol}_{5}(S) & =720 \operatorname{vol}_{5}(Z) \\
& =120 \operatorname{vol}_{5}\left(Z_{2}\right)=48 \operatorname{vol}_{5}\left(Z_{3}\right)=36 \operatorname{vol}_{5}\left(Z_{4}\right)
\end{aligned}
$$

\subsection{Extending to Infinity}

Let us start with the easiest case and consider a doubly asymptotic 5-orthoscheme $R=p_{0} \cdots p_{5}$ with vertices $p_{0}, \ldots, p_{5}$ and with graph

$$
\Sigma(R) \quad: \quad \bullet \frac{\alpha_{1}}{2} \circ \frac{\alpha_{2}}{2} \circ \frac{\alpha_{3}}{2} \circ \frac{\alpha_{4}}{\circ} \circ \frac{\alpha_{5}}{\bullet}
$$

The bi-asymptoticity of $R$ can be expressed according to (cf. (26))

$$
\tan \alpha_{1} \tan \alpha_{2}=\tan \alpha_{4} \tan \alpha_{5}
$$

Now, prolong the geodesic half-line $p_{0} p_{1}$ defined by $p_{0}$ and $p_{1}$ beyond $p_{1}$ in order to obtain a geodesic line $l$. Denote by $q_{0} \in \partial \mathbb{H}^{5}$ the limiting point associated with $l$. Let $H$ be the hyperplane through $q_{0}$ orthogonal to the line defined by $p_{0}$ and $p_{5}$, and let $q_{i}:=H \cap p_{0} p_{i+1}$ for $i=1, \ldots, 4$. Set $q_{5}:=p_{0}$ and consider the convex hull

$$
R_{k}:=q_{0} \cdots q_{k-1} p_{k} \cdots p_{5}, \quad 0 \leq k \leq 6 .
$$

Obviously, $R=R_{0}$, and write $Q:=R_{6}$. Then, by [3, Theorem (2.6) and Sect. 6], each $R_{k}$ is a doubly asymptotic 5-orthoscheme which takes part of the scissors congruence relation

$$
[R]=-\sum_{k=1}^{p}\left[R_{k}\right]+\sum_{k=p+1}^{6}\left[R_{k}\right],
$$

where the index $p \in[1,5]$ depends on the measures of the dihedral angles $\alpha_{1}, \ldots, \alpha_{5}$ of $R$. For example, if the dihedral angle $\alpha_{1}<\pi / 4$ (resp. $\alpha_{1}>\pi / 4$ ), then the foot point $q_{1}$ of the perpendicular from $q_{0}$ to the half-line $p_{0} p_{2}$ lies inside of $R$ (resp. outside of $R$ ), a fact which we denote by $q_{1} \in R$ (resp. $q_{1} \notin R$ ); see also Fig. 5. By 
the identity (33) and the parabolicity condition (2), it is easy to see that $R$ is isometric to $R_{1}$ and that $Q$ is isometric to $R_{5}$ (cf. dihedral angle distribution in Fig. 1). The graph of $Q$ is given by

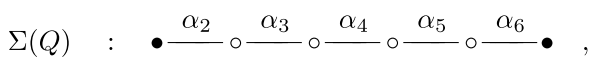

where $\left.\alpha_{6} \in\right] 0, \frac{\pi}{2}\left[\right.$ is given by (24). Hence, if $\alpha_{1}<\pi / 4$ and $q_{1} \in R$, then $p=1$, and (34) turns into (cf. [10, (18)])

$$
2[R]=2[Q]+\left[R_{2}\right]+\left[R_{3}\right]+\left[R_{4}\right] .
$$

Now, denote the graphs of $R_{2}, R_{3}, R_{4}$ as follows (cf. also Fig. 1):

$$
\begin{aligned}
& \Sigma\left(R_{2}\right) \quad: \quad \bullet \varphi_{1} \circ \frac{x_{1}}{\circ} \circ \frac{y_{1}}{\circ} \circ \frac{\alpha_{4}}{x_{1}} \circ \frac{\alpha_{5}}{\alpha_{5}} \bullet \\
& \Sigma\left(R_{3}\right) \quad: \quad \bullet \frac{\alpha_{2}}{\alpha_{2}} \circ \frac{x_{2}}{\alpha_{2}} \circ \frac{y_{2}}{\alpha_{1}} \circ \stackrel{\alpha_{5}}{\bullet} \bullet \\
& \Sigma\left(R_{4}\right) \quad: \quad \bullet \frac{\alpha_{2}}{-} \circ \frac{\alpha_{3}}{\circ} \circ \stackrel{y_{3}}{\circ} \circ \frac{z_{2}}{-} \circ \stackrel{\varphi_{6}}{\longrightarrow} \text {, }
\end{aligned}
$$

where the parameter $\left.\varphi_{k} \in\right] 0, \frac{\pi}{2}[, k=1,6$, depends-by parallelism considerationson $\alpha_{k}$ as follows:

$$
\varphi_{k}:= \begin{cases}2 \alpha_{k}, & \text { if } \alpha_{k}<\frac{\pi}{4} \\ \pi-2 \alpha_{k}, & \text { if } \alpha_{k}>\frac{\pi}{4} .\end{cases}
$$

By the parabolicity conditions (2) and (33), the dihedral angles $x_{1}, y_{1}, z_{2}, y_{3}$ are easy to determine. As for $\left.x_{2} \in\right] 0, \frac{\pi}{2}$ [ and finally $p$ (cf. (34)), consider the different positions of $q_{1}$ and $q_{2}$ relative to $R$ inside the 3-faces $q_{0} q_{1} p_{2} p_{3}$ and $q_{0} q_{1} q_{2} p_{3}$ and the position of $q_{3}$ relative to $R$. It follows at once that

$$
x_{2}= \begin{cases}\alpha_{2}-x_{1}, & \text { if } q_{1} \in R \text { and } p=1 ; \\ \alpha_{2}+x_{1}, & \text { if } q_{1} \notin R \text { and } q_{2} \in R \text { and } p=2 ; \\ \pi-\left(\alpha_{2}+x_{1}\right), & \text { if } q_{1}, q_{2} \notin R \text { and } q_{3} \in R \text { and } p=3 ; \\ \pi-\left(\alpha_{2}+x_{1}\right), & \text { if } q_{1}, q_{2}, q_{3} \notin R \text { and } p=4 .\end{cases}
$$

We illustrate these dissections by providing several useful examples connecting members of the $W$-cycle with members of the $V$-cycle and-most importantly-the orthoscheme $Z_{4}$ (cf. (4)-(7), also Sect. 3.4 below).

Example 1 For the pseudo-Coxeter orthoscheme $R$ with graph (cf. (5))

$$
W_{2}=\Sigma(R) \quad: \quad \bullet \frac{5}{-} \circ \stackrel{\omega_{2}}{\omega_{1}} \circ \stackrel{\frac{5}{2}}{\circ} \stackrel{3}{\longrightarrow},
$$

one obtains

$$
W_{1}=\Sigma(Q) \quad: \quad \bullet \frac{\omega_{2}}{-} \circ \frac{\omega_{1}}{\frac{5}{2}} \circ \stackrel{3}{-} \circ \frac{3}{\longrightarrow}
$$


so that $q_{1}, q_{3} \in R$ by (37). Furthermore, by (9) and (38a), one derives that $p=1$ and

$$
\begin{aligned}
& V_{3}=\Sigma\left(R_{2}\right) \quad: \quad \bullet \frac{\frac{5}{2}}{\circ} \stackrel{3}{\longrightarrow} \circ \stackrel{5}{\frac{5}{2}} \circ \frac{3}{\longrightarrow} \bullet \\
& W_{1}=\Sigma\left(R_{3}\right) \quad: \quad \bullet \frac{\omega_{2}}{-} \circ \stackrel{\omega_{1}}{\longrightarrow} \stackrel{\frac{5}{2}}{\circ} \stackrel{3}{\longrightarrow} \circ \frac{3}{-} \bullet \\
& W_{1}=\Sigma\left(R_{4}\right) \quad: \quad \bullet \omega_{2} \circ \stackrel{\omega_{1}}{\longrightarrow} \stackrel{\frac{5}{2}}{\longrightarrow} \stackrel{3}{\longrightarrow} \circ \frac{3}{\longrightarrow}
\end{aligned}
$$

Hence, by (35), we deduce that

$$
2\left[W_{2}\right]=4\left[W_{1}\right]+\left[V_{3}\right] .
$$

Example 2 For the pseudo-Coxeter orthoscheme $R$ with graph

$$
W_{3}=\Sigma(R) \quad: \quad \bullet \frac{3}{\circ} \circ \stackrel{5}{\longrightarrow} \stackrel{\omega_{2}}{\longrightarrow} \circ \stackrel{\omega_{1}}{\longrightarrow} \stackrel{\frac{5}{2}}{\longrightarrow},
$$

one gets $q_{1} \notin R$ and

$$
W_{2}=\Sigma(Q) \quad: \quad \bullet \frac{5}{\circ} \circ \stackrel{\omega_{2}}{\circ} \stackrel{\omega_{1}}{\longrightarrow} \stackrel{\frac{5}{2}}{\circ} \stackrel{3}{\longrightarrow},
$$

so that $q_{3} \in R$ by (37). By (38b), we obtain $p=2$ and

$$
\begin{aligned}
W_{3} & =\Sigma\left(R_{2}\right) \quad: \bullet \frac{3}{5} \circ \frac{5}{5} \circ \frac{\omega_{2}}{\frac{5}{2}} \circ \frac{\omega_{1}}{3} \circ \frac{5}{5} \circ \frac{\frac{5}{2}}{\bullet} \bullet \\
V_{2} & =\Sigma\left(R_{3}\right) \quad: \bullet \frac{5}{5} \circ \omega_{2} \circ \omega_{1} \circ \frac{\frac{5}{2}}{} \circ \frac{3}{} \cdot \\
W_{2} & =\Sigma\left(R_{4}\right) \quad:
\end{aligned}
$$

Hence, by (34), we deduce that

$$
3\left[W_{3}\right]=3\left[W_{2}\right]+\left[V_{2}\right] .
$$

Example 3 For the pseudo-Coxeter orthoscheme $R$ with graph

$$
W_{4}=\Sigma(R) \quad: \quad \bullet \frac{3}{\circ} \circ \stackrel{3}{\circ} \circ \stackrel{5}{=} \stackrel{\omega_{2}}{=} \stackrel{\omega_{1}}{\longrightarrow},
$$

one gets $q_{1} \notin R$ and

$$
W_{3}=\Sigma(Q) \quad: \quad \bullet \frac{3}{\circ} \circ \stackrel{5}{\circ} \circ \frac{\omega_{2}}{\circ} \circ \frac{\omega_{1}}{} \circ \stackrel{\frac{5}{2}}{\bullet},
$$

so that $q_{3} \in R$ by (37). By (38b), we obtain $p=3$ and

$$
\begin{aligned}
& W_{4}=\Sigma\left(R_{2}\right) \quad: \bullet \frac{3}{3} \circ \frac{3}{3} \circ \frac{5}{5} \circ \frac{\omega_{2}}{5} \circ \frac{\omega_{1}}{\omega_{2}} \circ \frac{\omega_{1}}{\bullet} \\
& W_{4}=\Sigma\left(R_{3}\right) \quad: \bullet \frac{3}{5} \circ \frac{5}{5} \circ \frac{5}{2} \circ \frac{3}{5} \bullet . \\
& V_{1}=\Sigma\left(R_{4}\right) \quad: \quad \frac{5}{},
\end{aligned}
$$


Hence, by (34), we obtain

$$
4\left[W_{4}\right]=2\left[W_{3}\right]+\left[V_{1}\right] .
$$

Example 4 For the pseudo-Coxeter orthoscheme $R$ with graph

$$
V_{3}=\Sigma(R) \quad: \quad \bullet \frac{\frac{5}{2}}{\circ} \stackrel{3}{\longrightarrow} \circ \stackrel{5}{\circ} \stackrel{\frac{5}{2}}{\circ} \stackrel{3}{\longrightarrow},
$$

one gets $q_{1} \notin R$ and

$$
V_{1}=\Sigma(Q) \quad: \quad \cdot \frac{3}{-} \circ \stackrel{5}{\circ} \circ \stackrel{\frac{5}{2}}{\circ} \stackrel{3}{-} \stackrel{5}{\longrightarrow},
$$

so that $q_{3} \notin R$ by (37). By (38d), we obtain $p=4$ and, by (6) and (7),

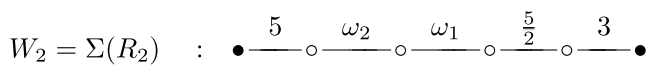

$$
\begin{aligned}
& Z_{4}=\Sigma\left(R_{3}\right) \quad: \quad \stackrel{3}{-} \circ \frac{\lambda}{2} \circ \stackrel{\lambda}{\lambda} \circ \stackrel{3}{-} \\
& W_{3}=\Sigma\left(R_{4}\right) \quad: \quad \stackrel{3}{-5} \circ \stackrel{5 \omega_{2}}{-} \stackrel{\omega_{1}}{\circ} \stackrel{\frac{5}{2}}{\bullet}
\end{aligned}
$$

By (34), we deduce that

$$
2\left[V_{1}\right]=2\left[V_{3}\right]+\left[W_{2}\right]+\left[W_{3}\right]+\left[Z_{4}\right]
$$

Remark 3 The extension to infinity as described by (34) can be easily generalised to the case when the vertex $p_{0}$ of $R$ is finite (respectively ultra-ideal). The orthoschemes $R_{k}, 1 \leq k \leq 5$, will be 2 -asymptotic, and the orthoscheme $R_{6}=Q$ will be simply asymptotic (respectively truncated with respect to $p_{0}$ ). More precisely, we obtain a dissection according to (34) where the graphs of $R_{1}, \ldots, R_{5}$ are as follows:

$$
\begin{aligned}
& \Sigma\left(R_{1}\right) \quad: \quad \bullet \frac{\varphi_{1}}{\varphi_{1}} \circ \frac{\varphi_{2}}{\circ} \circ \frac{\alpha_{3}}{\circ} \circ \frac{\alpha_{4}}{\alpha_{1}} \circ \frac{\alpha_{5}}{\bullet} \bullet \\
& \Sigma\left(R_{2}\right) \quad: \quad \frac{\psi_{1}}{2} \circ \frac{x_{1}}{2} \circ y_{1} \circ \frac{\alpha_{4}}{2} \circ \frac{\alpha_{5}}{\bullet} \text {. } \\
& \Sigma\left(R_{3}\right) \quad: \quad \bullet \frac{\alpha_{2}}{-} \circ x_{2} \circ \stackrel{y_{2}}{\longrightarrow} \circ \frac{z_{1}}{\alpha_{3}} \stackrel{\alpha_{5}}{-} \\
& \Sigma\left(R_{4}\right) \quad: \quad \bullet \frac{\alpha_{2}}{-} \circ \frac{\alpha_{3}}{\alpha_{3}} \circ \frac{y_{3}}{\circ} \circ \frac{z_{2}}{\alpha_{3}} \circ \stackrel{\psi_{2}}{\longrightarrow} \bullet \\
& \Sigma\left(R_{5}\right) \quad: \quad \bullet \frac{\alpha_{2}}{-} \circ \frac{\alpha_{3}}{\square} \circ \frac{\alpha_{4}}{\square} \circ \varphi_{3} \circ \stackrel{\varphi_{4}}{\longrightarrow} \bullet \text {, }
\end{aligned}
$$

where the parameters $\left.\varphi_{k} \in\right] 0, \frac{\pi}{2}\left[\right.$ as well as $x_{1}, y_{1}, y_{2}, z_{1}, z_{2}$ are determined by the parabolicity conditions (2) and (33), while

$$
\begin{aligned}
& \psi_{1}= \begin{cases}\alpha_{1}+\varphi_{1}, & \text { if } \alpha_{1}+\varphi_{1}<\frac{\pi}{2}, \\
\pi-\left(\alpha_{1}+\varphi_{1}\right), & \text { if } \alpha_{1}+\varphi_{1}>\frac{\pi}{2}\end{cases} \\
& \psi_{2}= \begin{cases}\alpha_{6}+\varphi_{4}, & \text { if } \alpha_{6}+\varphi_{4}<\frac{\pi}{2} \\
\pi-\left(\alpha_{6}+\varphi_{4}\right), & \text { if } \alpha_{6}+\varphi_{4}>\frac{\pi}{2}\end{cases}
\end{aligned}
$$


and

$$
x_{2}= \begin{cases}\varphi_{2}-x_{1}, & \text { if } q_{1} \in R \text { and } p=1 ; \\ \varphi_{2}+x_{1}, & \text { if } q_{1} \notin R \text { and } q_{2} \in R \text { and } p=2 ; \\ \pi-\left(\varphi_{2}+x_{1}\right), & \text { if } q_{1}, q_{2} \notin R \text { and } q_{3} \in R \text { and } p=3 ; \\ \pi-\left(\varphi_{2}+x_{1}\right), & \text { if } q_{1}, q_{2}, q_{3} \notin R \text { and } p=4 .\end{cases}
$$

We describe this case by giving some useful examples.

Example 5 For the pseudo-Coxeter orthoscheme $R$ with graph

$$
A=\Sigma(R) \quad: \quad \circ \frac{5}{\circ} \circ \frac{3}{\circ} \circ \stackrel{\frac{5}{2}}{\longleftarrow} \stackrel{5}{-} \stackrel{\frac{5}{2}}{\longrightarrow},
$$

one gets $q_{1} \notin R$ and

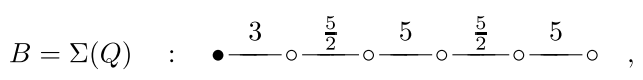

so that $q_{3} \in R$. Furthermore, one sees that $q_{2} \in R$ implying $p=2$. It follows that

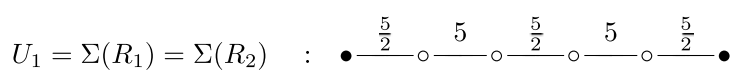

$$
\begin{aligned}
& V_{3}=\Sigma\left(R_{3}\right)=\Sigma\left(R_{4}\right)=\Sigma\left(R_{5}\right) \quad: \quad \stackrel{3}{\circ} \stackrel{\frac{5}{2}}{5} \div \frac{3}{3} \circ \frac{\frac{5}{2}}{\bullet} \text {. }
\end{aligned}
$$

By (34), we deduce that

$$
[A]=[B]+3\left[V_{3}\right]-2\left[U_{1}\right] .
$$

Example 6 For the pseudo-Coxeter orthoscheme $R$ with graph

$$
C=\Sigma(R) \quad: \quad \circ 5 \circ \frac{3}{-} \circ \stackrel{3}{\frac{5}{2}} \circ 5
$$

one gets $q_{1} \notin R$ and

$$
D=\Sigma(Q) \quad: \quad \cdot \frac{3}{2} \circ \frac{3}{\square} \circ \stackrel{\frac{5}{2}}{5} \stackrel{5}{\square} \circ,
$$

so that $q_{3} \in R$. Furthermore, one sees that $q_{2} \in R$ implying $p=2$. Using (6) and (7), one derives that

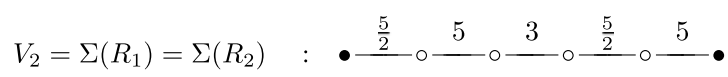

$$
\begin{aligned}
& W_{2}=\Sigma\left(R_{3}\right) \quad: \quad \cdot \frac{3}{3} \circ \stackrel{\frac{5}{2}}{\frac{\omega_{1}}{\lambda}} \circ \frac{\omega_{2}}{2 \lambda} \circ \frac{5}{\lambda} \text {. } \\
& Z_{3}=\Sigma\left(R_{4}\right) \quad: \quad \cdot 3 \circ \stackrel{3}{\longrightarrow} \circ \stackrel{\lambda}{2} \stackrel{2 \lambda}{\longrightarrow} \circ \stackrel{\lambda}{\longrightarrow} \text {. } \\
& W_{1}=\Sigma\left(R_{5}\right) \quad: \quad \cdot \frac{3}{3} \circ \stackrel{\frac{5}{2}}{\longrightarrow} \circ \frac{\omega_{1}}{\longrightarrow} \circ \stackrel{\omega_{2}}{\longrightarrow} \text {. }
\end{aligned}
$$


By (34), we deduce that

$$
[C]=[D]+\left[W_{1}\right]+\left[W_{2}\right]+\left[Z_{3}\right]-2\left[V_{2}\right] .
$$

The next two examples serve exclusively to illustrate the differences between the seemingly related ordinary orthoschemes belonging to (11) and satisfying (43), (44) and the truncated counterparts belonging to (12).

Example 7 For the pseudo-Coxeter orthoprism $R$ with graph

$$
\bar{A}=\Sigma(R) \quad: \quad \diamond \frac{\frac{5}{2}}{\circ} \stackrel{3}{\longrightarrow} \circ \stackrel{5}{\frac{5}{2}} \circ \frac{5}{\bullet},
$$

one gets

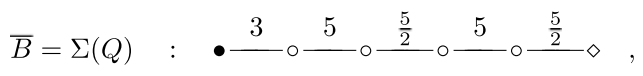

and $p=3$. Here, one derives

$$
[\bar{A}]=[\bar{B}]+\left[W_{3}\right]-\left[W_{2}\right]+\left[V_{1}\right]-\left[V_{2}\right]-\left[U_{2}\right] .
$$

Example 8 For the pseudo-Coxeter orthoprism $R$ with graph

$$
\bar{C}=\Sigma(R) \quad: \quad \diamond \frac{\frac{5}{2}}{\circ} \stackrel{3}{\circ} \circ \stackrel{3}{\circ} \stackrel{5}{\frac{5}{2}} \bullet,
$$

one gets

$$
\bar{D}=\Sigma(Q) \quad: \quad \bullet \frac{3}{2} \circ \frac{3}{\circ} \circ \stackrel{5}{\frac{5}{2}} \circ \stackrel{3}{\bullet} \diamond,
$$

so that $q_{3} \notin R$ implying $p=4$. Here, one derives

$$
[\bar{C}]=[\bar{D}]+\left[W_{4}\right]-\left[W_{1}\right]-\left[U_{1}\right]-\left[V_{2}\right]-\left[V_{3}\right] .
$$

Remark 4 Another version of extending to infinity is to consider an orthoscheme $R$ with vertex at infinity $p_{0}$ and with ultra-ideal vertex $p_{5}$ which, by truncation, leads to an asymptotic orthoprism with graph

$$
\Sigma(R) \quad: \quad \bullet \frac{\alpha_{1}}{\alpha_{2}} \circ \frac{\alpha_{3}}{\alpha_{3}} \circ \frac{\alpha_{4}}{\alpha_{5}} \diamond \frac{\alpha_{5}}{\diamond} .
$$

The corresponding case with finite vertex $p_{5}$ will not be considered here since the reversion process of Sect. 3.1 applied to the group (11) provides identical dissections. By extending to infinity the half-line $p_{0} p_{1}$, the limit point $q_{0} \in \partial \mathbb{H}^{5}$ together with the vertices $p_{1}, \ldots, p_{5}$ yields a polytope $R_{1}$ isometric to $R$. The hyperplane $H$ through $q_{0}$ and orthogonal to the line determined by $p_{0}, p_{5}$ provides as in (34) a dissection of $R$ by means of truncated orthoschemes $R_{k}=q_{0} \cdots q_{k-1} p_{k} \cdots p_{5}$ with $q_{i}=H \cap p_{0} p_{i+1}$, with the exception that the member $Q=R_{6}$ is a doubly asymptotic orthoscheme with graph

$$
\Sigma(Q) \quad: \quad \bullet \frac{\alpha_{2}}{\circ} \circ \frac{\alpha_{3}}{2} \circ \frac{\alpha_{4}}{2} \circ \frac{\alpha_{5}}{2} \circ \frac{\alpha_{6}}{\bullet},
$$


where $\alpha_{6}$ is given by (24). Hence,

$$
2[R]+\sum_{k=2}^{p}\left[R_{k}\right]=\sum_{k=p+1}^{5}\left[R_{k}\right]+[Q],
$$

where the sum of the left-hand side is void if $p=1$, and where the explicit description of $R_{2}, \ldots, R_{5}$ is based on standard non-Euclidean computations (see Sects. 3.1 and 3.2) yielding

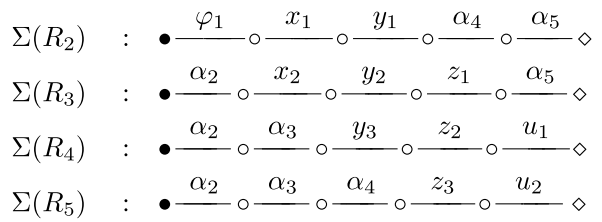

The angle $\varphi_{1}$ satisfies the condition (37), and the angle $x_{2}$ is subject to the conditions (38a)-(38d). The relation

$$
\tan x_{1}=\tan \alpha_{1} \tan \alpha_{2} \cot \varphi_{1}
$$

follows from comparing the hypotenuse of the vertex figures associated with $p_{3}$ in the 3 -faces $q_{0} q_{1} p_{2} p_{3}$ and $q_{0} p_{1} p_{2} p_{3}$, and the parameter $y_{1}$ is determined by the parabolicity condition (2). As for the remaining parameters, observe that

$$
y_{2}=\left\{\begin{array}{ll}
\pi-\left(y_{1}+y_{3}\right), & \text { if } p=1,4, \\
y_{1}-y_{3}, & \text { if } p=2, \\
y_{3}-y_{1}, & \text { if } p=3,
\end{array} \quad \text { and } \quad z_{2}= \begin{cases}\pi-\left(z_{1}+z_{3}\right), & \text { if } p=1,2, \\
z_{1}-z_{3}, & \text { if } p=3, \\
z_{3}-z_{1}, & \text { if } p=4 .\end{cases}\right.
$$

Indeed, the relation between the dihedral angles $y_{1}, y_{2}, y_{3}$ which are attached to the edge $q_{1} p_{4}$ in the 3 -faces $q_{1} p_{2} p_{3} p_{4}, q_{1} q_{2} p_{3} p_{4}, q_{1} q_{2} q_{3} p_{4}$ follows by their relative position in the cone $C$ with apex $p_{0}$ over the triangle $p_{2} p_{3} p_{4}$. The identity between $z_{1}, z_{2}, z_{3}$ follows by considering the edge $q_{2} p_{5}$ in $C$ in a similar fashion. By these identities and the parabolicity condition (2), it suffices to determine $z_{1}$ and $u_{1}$. First, observe that the edge length $p_{2} p_{3}$ (resp. $q_{2} q_{3}$ ) in the simply asymptotic 3-face $p_{0} p_{1} p_{2} p_{3}$ of $R$ (resp. $q_{0} q_{1} q_{2} q_{3}$ of $Q$ ) is given by

$$
\tanh p_{2} p_{3}=\cot \alpha_{1} \cot \alpha_{2} \quad\left(\text { resp. } \tanh q_{2} q_{3}=\cot \alpha_{2} \cot \alpha_{3}\right) \text {. }
$$

Next, consider the vertex figures at $p_{3}, q_{2}$ (resp. $p_{2}$ ) in the 3 -faces $q_{0} q_{1} q_{2} p_{3}$, $q_{2} p_{3} p_{4} p_{5}$ (resp. $\left.p_{2} p_{3} p_{4} p_{5}\right)$ of $R_{3}$. For the planar angles $\delta:=\angle\left(q_{0} p_{3} q_{2}\right), \sigma:=$ $\angle\left(p_{3} q_{2} p_{4}\right)$ and $\rho:=\angle\left(p_{3} p_{2} p_{4}\right)$ one easily deduces the identities

$$
\begin{array}{r}
\cos \delta=\cot \alpha_{2} \cot x_{2}, \quad \sin \delta=\frac{1}{\sinh q_{2} p_{3}}, \\
\cos \sigma=\frac{\cos z_{1}}{\sin \alpha_{5}}, \\
\cos \rho=\frac{\cos \alpha_{4}}{\sin \alpha_{5}}, \quad \tan \rho=\frac{\tanh p_{3} p_{4}}{\sinh p_{2} p_{3}},
\end{array}
$$


which together with (49) allow us to determine $z_{1}$. It remains to find $u_{1}$ which we perform in a similar way. Consider the planar angles $\zeta:=\angle\left(p_{4} q_{2} p_{5}\right)$ and $\xi:=\angle\left(q_{3} q_{2} p_{4}\right)$ in the 3 -face $q_{2} q_{3} p_{4} p_{5}$ of $R_{4}$. We obtain the trigonometrical identities

$$
\cos \zeta=\frac{\cos \alpha_{5}}{\sin z_{1}}, \quad \sin u_{1}=\frac{\tan \zeta}{\sin \xi}, \quad \frac{\tanh q_{2} q_{3}}{\cos \xi}=\tanh q_{2} p_{4}=\frac{\tanh q_{2} p_{3}}{\cos \sigma},
$$

which by means of (49) and (50) lead to $u_{1}$. Here are some illustrating and important examples.

Example 9 For the pseudo-Coxeter orthoprism $R$ with graph

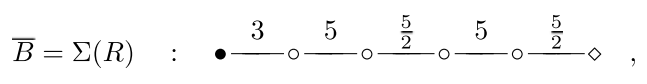

one gets $p=2$ and

$$
U_{2}=\Sigma(Q) \quad: \quad \bullet \frac{5}{2} \circ \stackrel{\frac{5}{2}}{\circ} \stackrel{5}{\longrightarrow} \circ \stackrel{\frac{5}{2}}{\circ} \stackrel{5}{\longrightarrow},
$$

as well as

$$
\begin{aligned}
& \bar{B}=\Sigma\left(R_{2}\right) \quad: \quad \bullet \frac{3}{5} \circ \frac{5}{5} \circ \stackrel{\frac{5}{2}}{5} \stackrel{5}{\longrightarrow} \circ \stackrel{\frac{5}{2}}{\diamond} \\
& \bar{A}=\Sigma\left(R_{3}\right)=\Sigma\left(R_{4}\right)=\Sigma\left(R_{5}\right) \quad: \quad \bullet \frac{5}{\circ} \stackrel{\frac{5}{2}}{\circ} \stackrel{5}{\longrightarrow} \stackrel{3}{\longrightarrow} \stackrel{\frac{5}{2}}{\diamond}
\end{aligned}
$$

Therefore,

$$
3[\bar{B}]=3[\bar{A}]+\left[U_{2}\right]
$$

Example 10 For the pseudo-Coxeter orthoprism $R$ with graph

$$
\bar{A}=\Sigma(R) \quad: \quad \bullet \frac{5}{\circ} \circ \stackrel{\frac{5}{2}}{\circ} \stackrel{5}{\longrightarrow} \circ \stackrel{3}{\frac{5}{2}} \diamond,
$$

one gets $p=1$ and

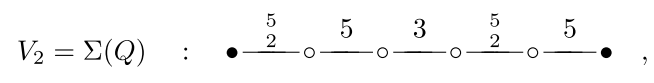

and

$$
\bar{C}=\Sigma\left(R_{2}\right)=\Sigma\left(R_{3}\right)=\Sigma\left(R_{4}\right)=\Sigma\left(R_{5}\right) \quad: \quad \bullet \frac{\frac{5}{2}}{\circ} \stackrel{5}{\square} \stackrel{3}{\longrightarrow} \circ \stackrel{3}{\frac{5}{2}} \diamond
$$

Hence,

$$
2[\bar{A}]=2[\bar{C}]+\left[V_{2}\right]
$$

Example 11 For the pseudo-Coxeter orthoprism $R$ with graph

$$
\bar{D}=\Sigma(R) \quad: \quad \bullet \frac{3}{2} \circ \stackrel{3}{-} \circ \frac{5}{\circ} \circ \stackrel{\frac{5}{2}}{\longrightarrow} \stackrel{3}{ } \diamond,
$$


one gets $p=3$ and

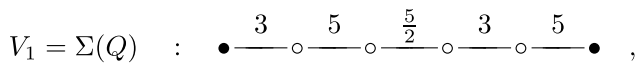

as well as

$$
\begin{aligned}
& \bar{D}=\Sigma\left(R_{2}\right)=\Sigma\left(R_{3}\right) \quad: \quad \bullet \frac{3}{3} \circ \frac{3}{5} \circ \frac{5}{\circ} \circ \frac{\frac{5}{2}}{5} \circ \frac{3}{\circ} \diamond \\
& \bar{B}=\Sigma\left(R_{4}\right)=\Sigma\left(R_{5}\right) \quad: \quad \bullet \frac{3}{\square} \circ \stackrel{\frac{5}{2}}{\circ} \circ \frac{5}{2} \diamond
\end{aligned}
$$

Therefore,

$$
4[\bar{D}]=2[\bar{B}]+\left[V_{1}\right] .
$$

It is interesting to observe the similarity of the scissors relations (52)-(54) to the ones in (20)-(22)!

\subsection{Extension and Polar Reflection}

Let $R=p_{0} \cdots p_{5} \subset \mathbb{H}^{5}$ denote an asymptotic orthoscheme with ideal vertex $p_{5}$ and ultra-infinite vertex $p_{0}$ which is truncated by the polar hyperplane $P_{0}$ associated with $p_{0}$. Denote the graph of $R$ by

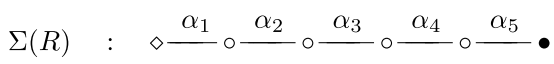

The hyperplane $P_{0}$ intersects the line determined by $p_{0}$ and $p_{1}$ at an ordinary point $\pi$, say. The two rays (of opposite orientation) $\pi p_{0}$ and $\pi p_{1}$ intersect the boundary $\partial \mathbb{H}^{5}$ in the ideal points $\bar{p}_{0}$ and $q_{0}$, say, which lie symmetrically with respect to $\pi$ and $P_{0}$. The following scissors procedure has first been described by Debrunner in the case of $\mathbb{H}^{3}$ (cf. [3, p. 145]). Consider as usual the hyperplane $H$ through $q_{0}$ and orthogonal to $p_{0} p_{5}$ providing the intersection points $q_{1}, \ldots, q_{4}$ on the edges $p_{0} p_{2}, \ldots, p_{0} p_{5}$. In the same way, consider the hyperplane $\bar{H}$ through $\bar{p}_{0}$ and orthogonal to $p_{0} p_{5}$ providing the intersection points $\bar{p}_{1}, \ldots, \bar{p}_{4}$ on the edges $p_{0} p_{2}, \ldots, p_{0} p_{5}$. Since the polar hyperplane $P_{0}$ cuts orthogonally all hyperplanes passing through $p_{0}$, it follows from the construction that the prism $Q=: q_{0} \cdots q_{4} P_{0}$ given by the convex hull of the set $\left\{q_{0}, \ldots, q_{4}, P_{0} \cap R\right\}$ is isometric to the prism $\frac{q}{R}=: \bar{p}_{0} \cdots \bar{p}_{4} P_{0}$ given by the convex hull of the set $\left\{\bar{p}_{0}, \ldots, \bar{p}_{4}, P_{0} \cap R\right\}$. The prism $\bar{R}$ has the orthoscheme $\bar{p}_{0} \cdots \bar{p}_{4}$ as top face and shares the base $P_{0} \cap R$ with $Q$ whose top face is given by the orthoscheme $q_{0} \cdots q_{4}$ (cf. Fig. 6).

For their graph, we obtain

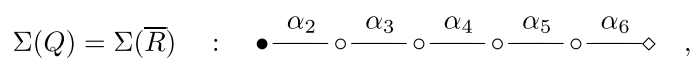

where $\alpha_{6}$ is given by the parabolicity identity (24). The following dissection is a natural consequence of our considerations. Consider the doubly asymptotic orthoschemes $\bar{R}_{k}:=\bar{p}_{0} \cdots \bar{p}_{k-1} p_{k} \cdots p_{5}, 1 \leq k \leq 5$. Then,

$$
[R]+[Q]=[R]+[\bar{R}]=\sum_{k=1}^{5}\left[\bar{R}_{k}\right],
$$




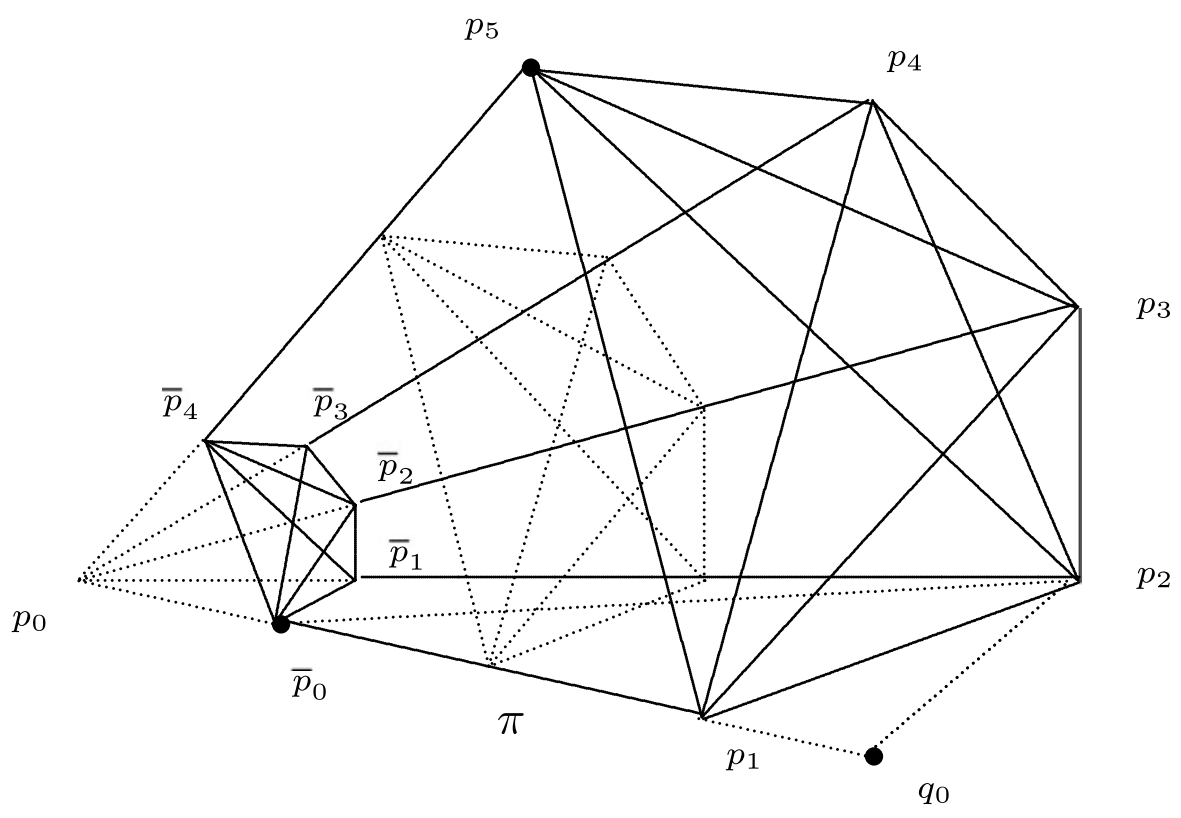

Fig. 6 Extension and polar reflection w.r.t. the polar hyperplane $P_{0}$ of $p_{0}$

where the graphs of $\bar{R}_{k}, 1 \leq k \leq 5$, are given by

$\Sigma\left(\bar{R}_{1}\right): \bullet \frac{u_{1}}{u_{2}} \circ \frac{x_{1}}{x_{2}} \circ \frac{\alpha_{3}}{y_{1}} \circ \frac{\alpha_{4}}{\alpha_{4}} \circ \frac{\alpha_{5}}{\alpha_{2}} \circ \frac{\alpha_{5}}{y_{2}} \bullet$
$\Sigma\left(\bar{R}_{2}\right): \bullet \frac{z_{1}}{x_{5}} \bullet \frac{\alpha_{5}}{v_{1}} \bullet$
$\Sigma\left(\bar{R}_{3}\right): \bullet \frac{\alpha_{2}}{x_{2}} \circ \frac{\alpha_{3}}{y_{3}} \circ \frac{z_{2}}{z_{2}} \circ \frac{\alpha_{2}}{v_{2}} \bullet$,
$\Sigma\left(\bar{R}_{5}\right): \bullet \frac{\alpha_{2}}{\alpha_{3}} \circ \frac{\alpha_{4}}{z_{3}} \circ \frac{v_{2}}{}$,

subject to the relations

$$
\begin{gathered}
u_{1}+u_{2}=\alpha_{1}, \quad v_{2}-v_{1}=\alpha_{5}, \\
x_{1}+x_{2}+x_{3}=\pi .
\end{gathered}
$$

In fact, the first two identities of (56) follow from the induced decompositions in the triangles $p_{0} p_{1} p_{2}$ and $p_{0} p_{3} p_{4}$, both truncated by $P_{0}$. The third identity follows from the fact that $x_{1}, x_{2}$ and $x_{3}$ are attached to the edge $\bar{p}_{0} p_{3}$ which is common to the 3-faces $\bar{p}_{0} p_{1} p_{2} p_{3}, \bar{p}_{0} \bar{p}_{1} p_{2} p_{3}$ and $\bar{p}_{0} \bar{p}_{1} \bar{p}_{2} p_{3}$ (cf. also Remark 4). These relations together with the parabolicity condition (2) allow to determine all parameters in (55). We illustrate the dissection (54) with some examples.

Example 12 For the pseudo-Coxeter orthoprism $R$ with graph

$$
\bar{A}=\Sigma(R) \quad: \quad \diamond \frac{\frac{5}{2}}{-} \circ \stackrel{3}{\longrightarrow} \circ \stackrel{5}{-\frac{5}{2}} \circ \stackrel{5}{\longrightarrow} \bullet,
$$


one gets

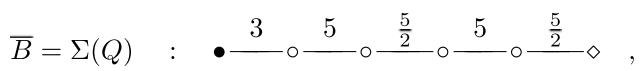

and

$$
\begin{aligned}
U_{2}=\Sigma\left(\bar{R}_{1}\right)=\Sigma\left(\bar{R}_{2}\right) \quad: \quad \bullet \frac{5}{3} \circ \frac{\frac{5}{2}}{\circ} \circ \frac{5}{5} \circ \frac{\frac{5}{2}}{\circ} \frac{5}{\bullet} \\
V_{1}=\Sigma\left(\bar{R}_{3}\right)=\Sigma\left(\bar{R}_{4}\right)=\Sigma\left(\bar{R}_{5}\right) \quad: \quad \bullet \frac{3}{\circ} \circ \frac{\frac{5}{2}}{} \circ \frac{3}{} \circ \stackrel{5}{ },
\end{aligned}
$$

Therefore,

$$
[\bar{A}]+[\bar{B}]=2\left[U_{2}\right]+3\left[V_{1}\right]
$$

Example 13 For the pseudo-Coxeter orthoprism $R$ with diagram

$$
\bar{C}=\Sigma(R) \quad: \quad \diamond \frac{\frac{5}{2}}{\circ} \stackrel{3}{\longrightarrow} \circ \stackrel{3}{\circ} \stackrel{5}{\stackrel{\frac{5}{2}}{\longrightarrow},},
$$

one gets

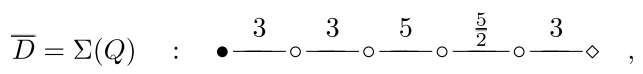

as well as

$$
\begin{aligned}
& V_{2}=\Sigma\left(\bar{R}_{1}\right)=\Sigma\left(\bar{R}_{2}\right) \quad: \quad \bullet \frac{5}{-} \circ \stackrel{\frac{5}{2}}{\circ} \stackrel{3}{\longrightarrow} \circ \frac{5}{5} \stackrel{\frac{5}{2}}{\bullet} \\
& W_{3}=\Sigma\left(\bar{R}_{3}\right) \quad: \quad \bullet \frac{3}{-} \circ \frac{5}{3} \circ \frac{\omega_{2}}{\lambda} \circ \omega_{1} \circ \stackrel{\frac{5}{2}}{~} \bullet \\
& Z_{3}=\Sigma\left(\bar{R}_{4}\right) \quad: \quad \bullet \frac{3}{3} \circ \stackrel{3}{-} \circ \stackrel{2 \lambda}{2} \circ \lambda \text {. } \\
& W_{4}=\Sigma\left(\bar{R}_{5}\right) \quad: \quad \bullet \frac{3}{\square} \circ \stackrel{5}{\square} \circ \stackrel{\omega_{2}}{\longrightarrow} \stackrel{\omega_{1}}{\longrightarrow}
\end{aligned}
$$

Therefore,

$$
[\bar{C}]+[\bar{D}]=2\left[V_{2}\right]+\left[W_{3}\right]+\left[W_{4}\right]+\left[Z_{3}\right] .
$$

The identities (57) and (58) should be compared with (43) and (44) of their nontruncated counterparts. The similarities are striking but the geometric background is mysterious.

\section{Volume Computations}

\subsection{A Simple Volume Formula}

In [11, Theorem 3], we derived an explicit formula for the volume of a doubly asymptotic hyperbolic 5-orthoscheme. Since the scissors congruence group $\mathcal{P}\left(\overline{\mathbb{H}^{5}}\right)$ of extended hyperbolic 5-space $\overline{\mathbb{H}^{5}}=\mathbb{H}^{5} \cup \partial \mathbb{H}^{5}$ is generated by doubly asymptotic orthoschemes, this result solves in principle the volume problem in hyperbolic 5-space. However, applying the formula to concrete cases such as (6) yields very complicated 
combinations of trilogarithmic values related to the weigths of (6). There is very little hope to simplify the expression by using the few known functional equations for the trilogarithm function involved. However, the volume formula simplifies drastically when restricting to doubly asymptotic orthoschemes $R=R(\alpha, \beta, \gamma)$ with graph of type (9), i.e.

$$
\Sigma(R) \quad: \quad \bullet \frac{\alpha}{\circ} \circ \frac{\beta}{\gamma} \circ \frac{\alpha}{\circ} \circ \frac{\beta}{\bullet}, \quad \cos ^{2} \alpha+\cos ^{2} \beta+\cos ^{2} \gamma=1
$$

For this case, we proved that (cf. [10, (4)])

$$
\begin{aligned}
\operatorname{vol}_{5}(R)= & \frac{1}{4}\left\{\Pi_{3}(\alpha)+\Pi_{3}(\beta)-\frac{1}{2} \Pi_{3}\left(\frac{\pi}{2}-\gamma\right)\right\} \\
& -\frac{1}{16}\left\{\pi_{3}\left(\frac{\pi}{2}+\alpha+\beta\right)+\pi_{3}\left(\frac{\pi}{2}-\alpha+\beta\right)\right\}+\frac{3}{64} \zeta(3),
\end{aligned}
$$

where the trilobachevsky function $\Pi_{3}(\omega)$ is related to the classical trilogarithm function

$$
\operatorname{Li}_{3}(z)=\sum_{r=1}^{\infty} \frac{z^{r}}{r^{3}}, \quad z \in \mathbb{C},
$$

by the identity (cf. [10, Sect. 2]; for $J_{2}(\omega)$, see (69))

$$
\Pi_{3}(\omega)=\frac{1}{4} \Re\left(\operatorname{Li}_{3}\left(e^{2 i \omega}\right)\right)=\frac{1}{4} \sum_{r=1}^{\infty} \frac{\cos (2 r \omega)}{r^{3}}=\frac{1}{4} \zeta(3)-\int_{0}^{\omega} \Pi_{2}(t) d t .
$$

The function $\pi_{3}(\omega)$ is even, $\pi$-periodic and satisfies the distribution law

$$
\frac{1}{m^{2}} \Omega_{3}(m \omega)=\sum_{r=0}^{m-1} \pi_{3}\left(\omega+\frac{r \pi}{m}\right) .
$$

In particular, we obtain the values

$$
\begin{aligned}
& \Omega_{3}(0)=\frac{1}{4} \zeta(3), \quad \Omega_{3}\left(\frac{\pi}{2}\right)=-\frac{3}{16} \zeta(3), \\
& \Omega_{3}\left(\frac{\pi}{5}\right)+\Omega_{3}\left(\frac{2 \pi}{5}\right)=-\frac{3}{25} \zeta(3) .
\end{aligned}
$$

Notice that formula (59) is a specialised 5-dimensional analogue of Lobachevsky's formula (see [9] and (68), for example) for a hyperbolic 3-orthoscheme $R=$ $R(\alpha, \beta, \gamma)$ with graph

$$
\Sigma(R) \quad: \quad \circ \frac{\alpha}{\beta} \circ \stackrel{\gamma}{\gamma} \circ \text { existing for } \cos \beta>\sin \alpha \sin \gamma
$$

In five dimensions, as an application of (59)-(62), one can determine easily the volumes of all members of the $V$-cycle (4), as first done in [10, Sect. 3.2]. In the sequel, we use the following notation. 
Notation For an orthoscheme $F$ with graph $\Sigma(F)$, put $f=\operatorname{vol}_{5}(\Sigma(F)):=\operatorname{vol}_{5}(F)$. Therefore,

$$
\begin{aligned}
& v_{1}=\operatorname{vol}_{5}\left(V_{1}\right)=\frac{1}{144}\left\{J_{3}\left(\frac{\pi}{5}\right)+\frac{\zeta(3)}{5}\right\} \approx 0.001998, \\
& v_{2}=\operatorname{vol}_{5}\left(V_{2}\right)=\frac{\zeta(3)}{1200} \approx 0.001002, \\
& v_{3}=\operatorname{vol}_{5}\left(V_{3}\right)=\frac{1}{144}\left\{-J_{3}\left(\frac{\pi}{5}\right)+\frac{2 \zeta(3)}{25}\right\} \approx 0.000339 .
\end{aligned}
$$

Furthermore, by using the dissection by extending to infinity as described in Sect. 3.3, we computed in [10, Sect. 3.2] the volumes of the two members of the $U$-cycle (3), and we obtained

$$
\begin{aligned}
& u_{1}=\operatorname{vol}_{5}\left(U_{1}\right)=\frac{1}{96} \pi_{3}\left(\frac{\pi}{5}\right) \approx 0.000493, \\
& u_{2}=\operatorname{vol}_{5}\left(U_{2}\right)=\frac{1}{96} \pi_{3}\left(\frac{\pi}{5}\right)+\frac{\zeta(3)}{800} \approx 0.001996 .
\end{aligned}
$$

\subsection{The Volumes of $A, B, C, D$ and $\bar{A}, \bar{B}, \bar{C}, \bar{D}$}

The simplices $A, B, C, D$ of the group (11) are simply asymptotic orthoschemes while the polytopes $\bar{A}, \bar{B}, \bar{C}, \bar{D}$ of the group (12) are simply asymptotic orthoprisms (or 1-truncated orthoschemes). For the graph $\Sigma$ of each of these polytopes $P$, we note

$$
\Sigma: \bullet \frac{\alpha_{1}}{\circ} \circ \frac{\alpha_{2}}{\circ} \circ \frac{\alpha_{3}}{\circ} \circ \frac{\alpha_{4}}{2} \circ \frac{\alpha_{5}}{\square,}
$$

where the vertex $\square$ represents either an ordinary vertex $\circ$ or an ultra-ideal vertex $\diamond$. Observe that a polytope of type (65) is described by four independent (acute) angle parameters uniquely up to isometry. Indeed, the parameters $\alpha_{2}, \ldots, \alpha_{5}$ satisfy the parabolicity relation (2). By the Schläfli volume differential (see e.g. [16, p. 118]), the volume of $P$ is - up to constants-equal to the simple integral of the differential

$$
\begin{aligned}
& \operatorname{vol}_{3}\left(\circ \stackrel{r}{-} \circ \stackrel{\alpha_{4}}{-} \circ \stackrel{\alpha_{5}}{-} \square\right) d \alpha_{1}+\operatorname{vol}_{3}\left(\bullet \stackrel{s}{-} \circ \stackrel{\alpha_{5}^{\prime}}{-} \circ \stackrel{\alpha_{5}}{u^{\prime}} \square\right) d \alpha_{2}+ \\
& \operatorname{vol}_{3}\left(\bullet \stackrel{t}{\circ} \circ u^{\prime} \circ \stackrel{u}{\square} \square\right) d \alpha_{3}+\operatorname{vol}_{3}\left(\bullet \frac{\alpha_{1}}{\alpha^{\prime}} \circ \stackrel{v^{\prime}}{\circ} \circ \square\right) d \alpha_{4}+ \\
& +\operatorname{vol}_{3}\left(\bullet \frac{\alpha_{1}}{\circ} \circ \frac{\alpha_{2}}{-} \circ \stackrel{\alpha_{2}^{\prime}}{\square} \square\right) d \alpha_{5}
\end{aligned}
$$

where $x^{\prime}:=\pi / 2-x$,

$$
\begin{aligned}
\tan r \tan \alpha_{1}= & \tan s \cot \alpha_{2}=\tan t \tan \alpha_{3}=\tan \theta_{1}=\frac{\sqrt{\sin ^{2} \alpha_{1} \sin ^{2} \alpha_{3}-\cos ^{2} \alpha_{2}}}{\cos \alpha_{1} \cos \alpha_{3}}, \\
\tan v \cot \alpha_{4} & =\tan u \tan \alpha_{3}=\tan \theta_{2}=\frac{\sqrt{\sin ^{2} \alpha_{3} \sin ^{2} \alpha_{5}-\cos ^{2} \alpha_{4}}}{\cos \alpha_{3} \cos \alpha_{5}},
\end{aligned}
$$


and where the integrands are given by the analytic expression (cf. [9, Theorem II])

$$
\begin{gathered}
\operatorname{vol}_{3}\left(\circ \stackrel{x}{\circ} \stackrel{y}{\circ} \circ \frac{z}{\square}\right)=\frac{1}{4}\left\{\mathrm{JI}_{2}(x+\theta)-\mathrm{J}_{2}(x-\theta)+\mathrm{JI}_{2}\left(\frac{\pi}{2}+y-\theta\right)+\right. \\
\left.+\mathrm{J}_{2}\left(\frac{\pi}{2}-y-\theta\right)+\mathrm{J}_{2}(z+\theta)-\mathrm{J}_{2}(z-\theta)+2 \mathrm{~J}_{2}\left(\frac{\pi}{2}-\theta\right)\right\},
\end{gathered}
$$

with

$$
\tan \theta=\frac{\sqrt{\cos ^{2} y-\sin ^{2} x \sin ^{2} z}}{\cos x \cos z}
$$

and the classical Lobachevsky function

$$
J_{2}(\omega)=\frac{1}{2} \Im\left(\operatorname{Li}_{2}\left(e^{2 i \omega}\right)\right)=\frac{1}{2} \sum_{r=1}^{\infty} \frac{\sin (2 r \omega)}{r^{2}}=-\int_{0}^{\omega} \log |2 \sin t| d t .
$$

In particular, in the limiting case, one obtains

$$
\operatorname{vol}_{3}\left(\bullet \stackrel{x}{\circ} \stackrel{y}{\circ} \stackrel{y^{\prime}}{\square}\right)=\frac{1}{4}\left\{\mathrm{~J}_{2}\left(\frac{\pi}{2}+x-y\right)-\mathrm{J}_{2}\left(\frac{\pi}{2}+x+y\right)+2 \mathrm{~J}_{2}(y)\right\} .
$$

Formulas (66)-(69) furnish evidence that the computation of $\operatorname{vol}_{5}(P)$ is very challenging and that the evaluation for particular examples does not provide simple expressions in the angle parameters. However, by means of the scissors relations (20)(57) and the elegant expressions (63), (64), we are able to determine the volumes of all members of the groups (11) and (12), and this without painful (and possibly unsuccessful) polylogarithmic calculations as just mentioned.

\section{Theorem I}

(a) The volumes of the simply asymptotic hyperbolic 5-orthoschemes $A, B, C, D$ as given by (11) are equal to

$$
\begin{aligned}
& a=\frac{\zeta(3)}{1200}-\frac{11}{576} \pi_{3}\left(\frac{\pi}{5}\right), \quad b=-\frac{\zeta(3)}{1200}+\frac{13}{576} \Omega_{3}\left(\frac{\pi}{5}\right), \\
& c=-\frac{\zeta(3)}{4800}+\frac{11}{1152} \Omega_{3}\left(\frac{\pi}{5}\right), \quad d=\frac{\zeta(3)}{1800}-\frac{5}{384} \pi_{3}\left(\frac{\pi}{5}\right) .
\end{aligned}
$$

(b) The volumes of the simply asymptotic hyperbolic 5-orthoprisms $\bar{A}, \bar{B}, \bar{C}, \bar{D}$ as given by (12) are equal to

$$
\begin{aligned}
& \bar{a}=\frac{\zeta(3)}{320}+\frac{11}{576} \pi_{3}\left(\frac{\pi}{5}\right), \quad \bar{b}=\frac{17 \zeta(3)}{4800}+\frac{13}{576} \Omega_{3}\left(\frac{\pi}{5}\right), \\
& \bar{c}=\frac{13 \zeta(3)}{9600}+\frac{11}{1152} \Omega_{3}\left(\frac{\pi}{5}\right), \quad \bar{d}=\frac{61 \zeta(3)}{28800}+\frac{5}{384} \pi_{3}\left(\frac{\pi}{5}\right) .
\end{aligned}
$$


Proof In order to verify the values in (a), combine the scissors equations (20) and (43) and derive $b=\frac{7}{6} u_{1}-\frac{3}{2} v_{3}$ and $a=-\frac{5}{6} u_{1}+\frac{3}{2} v_{3}$. Equation (21) allows us to conclude that $c=\frac{5}{12} u_{1}+\frac{1}{4} v_{2}-\frac{3}{4} v_{3}$. Finally, by (22), we obtain $d=-\frac{7}{12} u_{1}+v_{3}$. Now, plug (63) and (64) into the equations which yields (70).

For the proof of the identities (b), we proceed similarly and combine (51) and (57) in order to derive $\bar{b}=\frac{7}{6} u_{2}+\frac{3}{2} v_{1}$ and $\bar{a}=\frac{5}{6} u_{2}+\frac{3}{2} v_{1}$. Equation (52) then yields $\bar{c}=\frac{5}{12} u_{2}+\frac{3}{4} v_{1}-\frac{1}{4} v_{2}$. Together with (53), this implies that $\bar{d}=\frac{7}{12} u_{1}+v_{1}$. Again, by using the identities (63) and (64), the proof can be completed.

\subsection{The $W$-Cycle and the Ideal Regular 5-Simplex}

For some members of the $W$-cycle we can express their volume as rational linear combinations of three universal constants, namely by adjoining to Apéry's constant $\zeta(3)$ and the value $J_{3}\left(\frac{\pi}{5}\right) \simeq 0.0473419788$ the volume $\mu_{5}=\operatorname{vol}_{5}\left(S_{\text {reg }}^{\infty}(2 \lambda)\right)=$ $720 z \simeq 0.0575647377$ of an ideal regular 5-simplex (see (32)). We point out that the field $\mathbb{Q}(\sqrt{5})$, containing the golden ratio $\tau$, is the ground field associated with the (unique) arithmetic hyperbolic 5-orbifold of minimal volume (cf. [4] and [1]) while $\mu_{5}$ is the maximal volume among all hyperbolic 5-simplex volumes (cf. [6] and [13]). Of course, all three constants are certain trilogarithmic expressions. More precisely, $\mu_{5}$ can be expressed as

$$
\begin{aligned}
\mu_{5}= & 45 \int_{\lambda}^{\lambda_{0}}\left\{\Omega_{2}(\beta(t)+\theta(t))-\Omega_{2}(\beta(t)-\theta(t))\right. \\
& +\Omega_{2}\left(\frac{\pi}{6}-\theta(t)\right)-\Omega_{2}\left(\frac{\pi}{6}+\theta(t)\right)+\Omega_{2}\left(\frac{\pi}{3}+\theta(t)\right) \\
& \left.-\Omega_{2}\left(\frac{\pi}{3}-\theta(t)\right)+2 \Omega_{2}\left(\frac{\pi}{2}-\theta(t)\right)\right\} d t
\end{aligned}
$$

where $\lambda_{0}=\frac{1}{2} \arccos \frac{1}{5}, \beta(x)=\arccos \frac{\sin x}{\sqrt{4 \sin ^{2} x-1}}$ and $\theta(x)=\arctan \frac{\sqrt{2-5 \sin ^{2} x}}{\sin x}$.

In terms of $\zeta(3), \pi_{3}\left(\frac{\pi}{5}\right)$ and $\mu_{5}$, we obtain the following result.

Theorem II The volumes of the doubly asymptotic hyperbolic 5-orthoschemes $W_{1}, W_{2}, W_{3}, W_{4}$ of the $W$-cycle as given by (5) are equal to

$$
\begin{aligned}
& w_{1}=\frac{1}{144}\left\{\frac{5}{4} J_{3}\left(\frac{\pi}{5}\right)+\frac{3 \zeta(3)}{100}-\mu_{5}\right\}, \quad w_{2}=\frac{1}{72}\left\{\pi_{3}\left(\frac{\pi}{5}\right)+\frac{\zeta(3)}{20}-\mu_{5}\right\}, \\
& w_{3}=\frac{1}{72}\left\{J_{3}\left(\frac{\pi}{5}\right)+\frac{7 \zeta(3)}{100}-\mu_{5}\right\}, \quad w_{4}=\frac{1}{144}\left\{\frac{5}{4} \Omega_{3}\left(\frac{\pi}{5}\right)+\frac{3 \zeta(3)}{25}-\mu_{5}\right\} .
\end{aligned}
$$

Proof By means of the scissors identities (32), (40) and (42), we obtain the equation $w_{2}=v_{1}-\frac{1}{6} v_{2}-v_{3}-\frac{1}{72} \mu_{5}$. Then, (39) yields $w_{1}=\frac{1}{2} v_{1}-\frac{1}{12} v_{2}-\frac{3}{4} v_{3}-\frac{1}{144} \mu_{5}$. By using again (40), we obtain $w_{3}=v_{1}+\frac{1}{6} v_{2}-v_{3}-\frac{1}{72} \mu_{5}$. Finally, by (41), we deduce 
that $w_{4}=\frac{3}{4} v_{1}+\frac{1}{12} v_{2}-\frac{1}{2} v_{3}-\frac{1}{144} \mu_{5}$. The results then follow with the help of (63) and (64).

Remark 5 In [15, pp. 269-270], L. Schläfli considered two so-called periods of spherical 3-orthoschemes $R(\alpha, \beta, \gamma)$ with angle parameters $\frac{\pi}{3}, \frac{\pi}{3}, \frac{\pi}{3}, \lambda, 2 \lambda, \lambda$ (respectively $\left.\frac{\pi}{5}, \frac{\pi}{3}, \frac{\pi}{3}, \frac{2 \pi}{5} \omega_{1}=\frac{\pi}{3}-\lambda, \omega_{2}=\frac{2 \pi}{3}-\lambda\right)$ and deduced the values

$$
\begin{gathered}
\operatorname{vol}_{3}\left(R\left(\frac{\pi}{3}, \frac{\pi}{3}, \lambda\right)\right)=\frac{3 \pi}{4} \lambda-\frac{\pi^{2}}{60}, \\
\operatorname{vol}_{3}\left(R\left(\frac{\pi}{3}, \lambda, 2 \lambda\right)\right)=3 \pi \lambda-\frac{\pi^{2}}{15}, \\
\operatorname{vol}_{3}(R(\lambda, 2 \lambda, \lambda))=\frac{\pi}{2} \lambda-\frac{\pi^{2}}{10}, \\
\operatorname{vol}_{3}\left(R\left(\frac{\pi}{3}, \frac{2 \pi}{5}, \omega_{1}\right)\right)=-\frac{3 \pi}{4} \lambda+\frac{43 \pi^{2}}{2400}, \\
\operatorname{vol}_{3}\left(R\left(\frac{2 \pi}{5}, \omega_{1}, \omega_{2}\right)\right)=-\frac{\pi}{4} \lambda-\frac{391 \pi^{2}}{7200}, \\
\operatorname{vol}_{3}\left(R\left(\omega_{1}, \omega_{2}, \frac{\pi}{5}\right)\right)=-\frac{\pi}{4} \lambda-\frac{401 \pi^{2}}{7200}, \\
\operatorname{vol}_{3}\left(R\left(\omega_{2}, \frac{\pi}{5}, \frac{2 \pi}{5}\right)\right)=-\frac{3 \pi}{4} \lambda-\frac{53 \pi^{2}}{2400} .
\end{gathered}
$$

Observe that $\zeta(2)=\pi^{2} / 6$ and that the totally orthogonal regular 3-simplex has volume $\pi^{2} / 8$ which tops $\lambda \pi / 2$. The results in (73) are based—among other things—on the reduction principle for even dimensional (spherical) simplices. This important tool is not applicable when computing the volumes of the $W$-cycle!

Acknowledgements Partially supported by Schweizerischer Nationalfonds 200020-121506 and 200021131967.

\section{References}

1. Belolipetsky, M., Emery, V.: On volumes of arithmetic quotients of $\mathrm{PO}(n, 1), n$ odd. Preprint. arXiv:1001.4670. To appear in Proc. Lond. Math. Soc.

2. Coxeter, H.S.M.: Regular Polytopes. Dover, New York (1973)

3. Debrunner, H.E.: Dissecting orthoschemes into orthoschemes. Geom. Dedic. 33, 123-152 (1990)

4. Emery, V.: Du volume des quotients arithmétiques de l'espace hyperbolique. Ph.D. thesis no. 1648, Université de Fribourg (2009)

5. Gromov, M.: Hyperbolic manifolds according to Thurston and Jørgensen. Séminaire Bourbaki, vol. 22 (1979-1980). Exposé no. 546

6. Haagerup, U., Munkholm, H.J.: Simplices of maximal volume in hyperbolic $n$-space. Acta Math. 147, $1-12(1981)$

7. Hild, T.: The cusped hyperbolic orbifolds of minimal volume in dimensions less than ten. J. Algebra 313, 208-222 (2007)

8. Im Hof, H.-C.: Napier cycles and hyperbolic Coxeter groups. Bull. Soc. Math. Belg., Sér. A 42, 523545 (1990) 
9. Kellerhals, R.: On the volume of hyperbolic polyhedra. Math. Ann. 285, 541-569 (1989)

10. Kellerhals, R.: On the volumes of hyperbolic 5-orthoschemes and the trilogarithm. Comment. Math. Helv. 67, 648-663 (1992)

11. Kellerhals, R.: Volumes in hyperbolic 5-space. Geom. Funct. Anal. 5, 640-667 (1995)

12. Milnor, J.: How to compute volume in hyperbolic space. In: Geometry, Collected Papers, vol. 1. Publish or Perish, Berkeley (1994)

13. Peyerimhoff, N.: Simplices of maximal volume or minimal total edge length in hyperbolic space. J. Lond. Math. Soc., II Ser. 66, 753-768 (2002)

14. Ratcliffe, J.G.: Foundations of Hyperbolic Manifolds. Springer, New York (1994)

15. Schläfli, L.: Theorie der vielfachen Kontinuität. In: Gesammelte Mathematische Abhandlungen, vol. I. Birkhäuser, Basel (1950)

16. Vinberg, E.B.: Geometry II: Spaces of Constant Curvature. Encyclopaedia of Mathematical Sciences, vol. 29. Springer, Berlin (1993) 